quatrième série-tome $41 \quad$ fascicule $1 \quad$ janvier-février 2008

$$
\begin{aligned}
& \text { ANNALES } \\
& \text { SCIENTIFIQUES } \\
& \text { de } \\
& \text { L'ECOLE } \\
& \text { NORMALE } \\
& \text { SUPÉRIEURE }
\end{aligned}
$$

Jeremy KAHN \& Mikhail LYUBICH
A priori bounds for quadratic polynomials 
Ann. Scient. Éc. Norm. Sup.

$4^{\text {e }}$ série, t. 41,2008, p. 57 à 84

\title{
A PRIORI BOUNDS FOR SOME INFINITELY RENORMALIZABLE QUADRATICS: II. DECORATIONS
}

\author{
By Jeremy KAHN and Mikhail LYUBiCH
}

\begin{abstract}
A decoration of the Mandelbrot set $M$ is a part of $M$ cut off by two external rays landing at some tip of a satellite copy of $M$ attached to the main cardioid. In this paper we consider infinitely renormalizable quadratic polynomials satisfying the decoration condition, which means that the combinatorics of the renormalization operators involved is selected from a finite family of decorations. For this class of maps we prove a priori bounds. They imply local connectivity of the corresponding Julia sets and the Mandelbrot set at the corresponding parameter values.
\end{abstract}

RÉsumÉ. - Une décoration de l'ensemble de Mandelbrot $M$ est une partie de $M$ découpée par deux rayons externes aboutissant à la pointe d'une petite copie de $M$ attachée à la cardioïde principale. Dans cet article nous considérons des polynômes quadratiques infiniment renormalisables qui satisfont à la condition de décoration, à savoir que la combinatoire des opérateurs de renormalisation mis en jeu est sélectionnée à partir d'une famille finie de décorations. Pour cette classe d'applications, nous donnons des bornes a priori. Ces bornes impliquent la connexité locale des ensembles de Julia correspondants et celle de l'ensemble de Mandelbrot aux paramètres correspondants.

\section{Introduction}

A decoration of the Mandelbrot set $M$ (called also a Misiurewicz limb) $\mathcal{L}$ is a part of $M$ cut off by two external rays landing at some tip of a satellite copy of $M$ attached to the main cardioid, see Figure 1.1 (see $\$ 2.1$ for the precise dynamical definition). In this paper we consider infinitely renormalizable quadratic polynomials satisfying the decoration condition, which means that the combinatorics of the renormalization operators involved is selected from a finite family of decorations $\mathcal{L}_{k}$. (For instance, real infinitely renormalizable maps satisfy a decoration condition if and only if none of the renormalizations is of doubling type.)

An infinitely renormalizable quadratic map $f$ is said to have a priori bounds if its renormalizations can be represented by quadratic-like maps $R^{n} f: U_{n} \rightarrow V_{n}$ with $\bmod \left(V_{n} \backslash U_{n}\right) \geq$ $\varepsilon>0, n=1,2 \ldots$ 


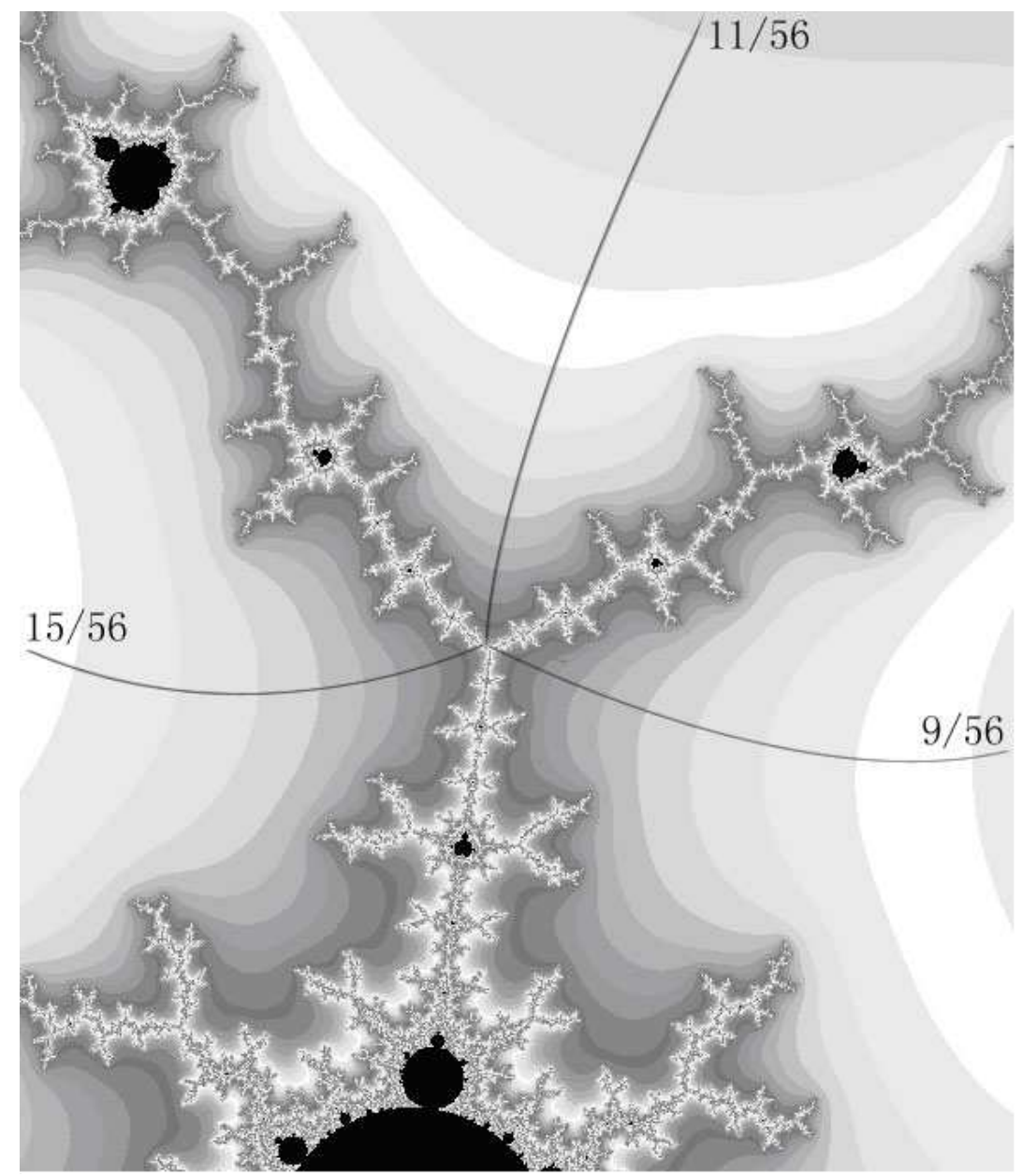

Figure 1.1. Two decorations of the Mandelbrot set. The little Mandelbrot sets inside specify renormalization combinatorics of type $(3,1)$.

Our goal is to prove the following result:

Main Theorem. - Infinitely renormalizable quadratic maps satisfying the decoration condition have a priori bounds.

By [9], this implies:

COROLLARY 1.1. - Let $f_{c}: z \mapsto z^{2}+c$ be an infinitely renormalizable quadratic map satisfying the decoration condition. Then the Julia set $J\left(f_{c}\right)$ is locally connected, and the Mandelbrot set $M$ is locally connected at $c$.

In this paper we will deal only with the case of sufficiently high periods: 
Theorem 1.2. - Given finitely many decorations $\mathcal{L}_{k}$, there exists a p such that any infinitely renormalizable quadratic map satisfying the decoration condition with decorations $\mathcal{L}_{k}$ and renormalization periods $p \geq p$ has a priori bounds.

The complementary case of "bounded combinatorics" is dealt with in [6].

REMARK 1.1. - Theorem 1.2 sounds similar in spirit to the a priori bounds of [9]. However, the "high type" condition of [9] is stronger than the above high period condition, while the "secondary limb condition" of [9] is weaker than the decoration condition. Also, our proof of Theorem 1.2 is compatible with the proof of [6], so that they can be combined into the Main Theorem.

Let us now outline the structure of the paper.

In the next section, $\S 2$, we will describe a necessary combinatorial set-up in the framework of the Yoccoz puzzle. Besides a well-known material, it includes the construction of the modified principal nest from [7] needed for dealing with maps of "high type".

In $\S 3$ we summarize necessary information about pseudo-quadratic-like maps defined in [6], and introduce a pseudo-puzzle by applying the "pseudo-functor" to the puzzle. In this way we make domains of the return maps more canonical, which spares us from the need to control geometry of external rays.

From now on, the usual puzzle will serve only as a combinatorial frame, while all the geometric estimates will be made on the pseudo-puzzle. This is needed for this paper per se, as well as for making connection to the case of bounded combinatorics [6]. Only at the last moment (\$5.7) we return back to the standard quadratic-like context.

In $\S 4$ we formulate the analytical results of [8], the Quasi-Additivity Law and the Covering Lemma, in the pseudo context. They will be our main analytical tools.

In $\S 5$ we prove the main results of the paper. To prove a priori bounds, we show that if some renormalization has a small modulus, then this modulus will improve on some deeper level. The main place where the decoration condition plays the role is on the top of the puzzle, when we compare the modulus of the first annulus of the pseudo-puzzle to the modulus of the original pseudo-quadratic-like map.

REMARK 1.2. - Strictly speaking, bounded combinatorics treated in [6] and high combinatorics treated by Theorem 1.2 do not cover the oscillating combinatorial types. However, these theorems follow from results on moduli improving, that together cover everything.

REMARK 1.3. - Our proof of a priori bounds (Main Theorem) applies without changes in the case of unicritical maps of higher degree. However, the proof of MLC at the corresponding parameters (Corollary 1.1) given in [9] exploits some special geometric features of quadratic maps. In [3] part of [9] is combined with a new method developed in [2] to prove Corollary 1.1 in the higher degree case as well. 


\subsection{Terminology and notation}

$\mathbb{N}=\{1,2, \ldots\}$ is the set of natural numbers; $\mathbb{Z}_{\geq 0}=\mathbb{N} \cup\{0\} ; \mathbb{D}=\{z:|z|<1\}$ is the unit disk, and $\mathbb{T}$ is the unit circle;

$\mathbb{A}(r, R)=\{z: r<|z|<R\}$ is the annulus of modulus $\frac{1}{2 \pi} \log (R / r)$;

$\Pi(h)=\{z \mid 0<\Im z<h\}$ is the horizontal strip.

A topological disk means a simply connected domain in some Riemann surface $S$. A continuum $K$ is a connected closed subset in $S$. It is called full if all components of $S \backslash K$ are unbounded. We say a subset $K$ of a plane is an FJ-set (for "filled Julia set") if $K$ is compact, connected, and full.

We let $\operatorname{orb}(z) \equiv \operatorname{orb}_{g}(z)=\left(g^{n} z\right)_{n=0}^{\infty}$ be the orbit of $z$ under a map $g$.

Given a map $g: U \rightarrow V$ and an open topological disk $D \subset V$, components of $g^{-1}(D)$ are called pullbacks of $D$ under $g$. If the disk $D$ is closed, we define pullbacks of $D$ as the closures of the pullbacks of int $D$. ${ }^{(1)}$ In either case, given a connected set $X \subset g^{-1}$ (int $D$ ), we let $g^{-1}(D) \mid X$ be the pullback of $D$ containing $X$.

We let $x \oplus y=\left(x^{-1}+y^{-1}\right)^{-1}$ be the harmonic sum of $x$ and $y$ (it is conjugate to the ordinary sum by the inversion map $\left.x \mapsto x^{-1}\right)$. Similarly, $x \ominus y=\left(x^{-1}-y^{-1}\right)^{-1}$ stands for the harmonic difference.

\subsection{Acknowledgement}

We thank Tao Li for making Figure 1.1. This work has been partially supported by the NSF, NSERC, the Guggenheim and Simons Foundations. Part of it was done during the authors' visit to the IMS at Stony Brook and the Fields Institute in Toronto. We are thankful to all these Institutions and Foundations.

\section{Yoccoz puzzle, decorations, and the Modified Principal Nest}

Let $\left(f_{\lambda}: U_{\lambda}^{\prime} \rightarrow U_{\lambda}\right)$ be a quadratic-like family over a disk $\Lambda \subset \mathbb{C}$. Assume that this family is good enough (proper and unfolded), so that the associated Mandelbrot set $M=M\left(f_{\lambda}\right)$ is canonically homeomorphic to the standard Mandelbrot set (see [4]). In fact, most of the time we will be dealing with a single map $f=f_{\lambda}$ from our family, so that we will usually suppress the label $\lambda$ in the notation. (We need a one parameter family only to introduce different combinatorial types of the maps under consideration.)

We assume that the domains $U^{\prime}$ and $U$ are smooth disks, $f$ is even, and we normalize $f$ so that 0 is its critical point.

We let $U^{m}=f^{-m}(U)$. The boundary of $U^{m}$ is called the equipotential of level $m$.

(1) Note that the pullbacks of a closed disk $D$ can touch one another, so they are not necessarily connected components of $g^{-1}(D)$.

$4^{\mathrm{e}}$ SÉRIE - TOME $41-2008-\mathrm{N}^{\mathrm{o}} 1$ 


\subsection{Top of the Yoccoz puzzle and decorations}

By means of straightening, we can define external rays for $f$. They form a foliation of $V \backslash K(f)$ orthogonal to the equipotential $\partial U$. The map $f$ has one non-dividing fixed point $\beta$ (landing point of the external ray with angle 0 ), and one dividing fixed point $\alpha$. There are $\mathbf{q}>1$ external rays $\mathcal{R}_{i}$ landing at $\alpha$ which are cyclically permuted by the dynamics with rotation number $\mathbf{p} / \mathbf{q}$, see [12] ( $\mathbf{p} / \mathbf{q}$ is also called the combinatorial rotation number of $\alpha$ ). These rays divide $U$ into $\mathbf{q}$ (closed) topological disks $Y_{i}^{0}$ called the Yoccoz puzzle pieces of depth 0 . Let $Y^{0} \equiv Y_{0}^{0}$ stand for the critical puzzle piece, i.e., the one containing 0 .

Let us consider $2 \mathbf{q}$ rays of $f^{-1}\left(\cup \mathcal{R}_{i}\right)$. They divide $U^{\prime}$ into $2 \mathbf{q}-1$ (closed) disks called Yoccoz puzzle pieces of depth 1 . Let $Y^{1}$ stand for the critical puzzle piece of depth 1 . There are also $\mathbf{q}-1$ puzzle pieces $Y_{i}^{1}$ of depth 1 contained in the corresponding off-critical pieces of depth 0 . All other puzzle pieces of depth 1 will be denoted $Z_{i}^{1}$. They are attached to the symmetric point $\alpha^{\prime}=-\alpha$.

The puzzle pieces will be labeled in such a way that $f\left(Y_{i}^{1}\right)=Y_{i+1}^{0}, i=0, \ldots, \mathbf{q}-1$, and $Z_{i}^{1}=-Y_{i}^{1}$. We let

$$
L=\bigcup_{i=1}^{\mathbf{q}-1} Y_{i}^{1} ; \quad R=-L=\bigcup_{i=1}^{\mathbf{q}-1} Z_{i}^{1} .
$$

Puzzle pieces $Y_{j}^{m}$ of depth $m$ are pullbacks of $f^{-m}\left(Y_{i}^{0}\right)$. They tile the neighborhood of $K(f)$ bounded by the equipotential $\partial U^{m}$. Each of them is bounded by finitely many arcs of this equipotential and finitely many external rays of $f^{-m}\left(\mathcal{R}_{i}\right)$. If $f^{m}(0) \neq \alpha$, then there is one puzzle piece of depth $m$ that contains the critical point 0 . It is called critical and is labeled as $Y^{m} \equiv Y_{0}^{m}$. These pieces are nested around the origin:

$$
Y^{0} \supset Y^{1} \supset Y^{2} \cdots \ni 0 .
$$

Let us consider a puzzle piece $Y=Y_{i}^{m}$. Different arcs of $\partial Y$ meet at the corners of $Y$. The corners where two external rays meet will be called vertices of $Y$; they are $f^{m}$-preimages of $\alpha$. Let $K_{Y}=K(f) \cap Y$. It is a closed connected set that meets the boundary $\partial Y$ at its vertices. Moreover, the external rays meeting at a vertex $v \in \partial Y$ chop off from $K(f)$ a continuum $S_{Y}^{v}$, the component of $K(f) \backslash$ int $Y$ containing $v$.

The critical value $f^{\mathbf{q}}(0)$ belongs to the puzzle piece $Y^{0}$. If in fact it belongs to $Y^{1}$ then the map $Y^{\mathbf{q}+1} \rightarrow Y^{1}$ is a double branched covering. It is not a quadratic-like map, though, since the boundaries of $Y^{1}$ and $Y^{\mathbf{q}+1}$ overlap over four external rays landing at $\alpha$ and $\alpha^{\prime}$. However, by slight "thickening" of the domain of this map (see [11]), it can be turned into a quadratic-like map $g$ such that

$$
K(g)=\left\{z: f^{\mathbf{q} m} z \in Y^{1}, m=0,1,2, \ldots\right\} .
$$

The map $f$ is called satellite renormalizable (or, immediately renormalizable) if the Julia set $K(g)$ is connected, i.e., if the critical point never escapes $Y^{1}$ :

$$
f^{\mathbf{q} m}(0) \in Y^{1}, \quad m=0,1,2 \ldots
$$

The set of immediately renormalizable parameter values (with a given combinatorial rotation number $\mathbf{p} / \mathbf{q}$ ) assembles a satellite copy $M_{\mathbf{p} / \mathbf{q}}$ of $M$ attached to the main cardioid at the parabolic point with rotation number $\mathbf{p} / \mathbf{q}$. The parameters $t \in M_{\mathbf{p} / \mathbf{q}}$ for which the critical point eventually lands at $\alpha$ (i.e., $f_{t}^{\mathbf{q} n}=\alpha^{\prime}$ for some $n \in \mathbb{N}$ ) are called the tips of $M_{\mathbf{p} / \mathbf{q}}$. 
If $f$ is not satellite renormalizable, then there exists an $n \in \mathbb{N}$ such that $f^{\mathbf{q n}}(0)$ belongs to some puzzle piece int $Z_{\boldsymbol{\kappa}}^{1}$. Let $\mathbf{n}$ be the smallest such $n$. In this case, we let

$$
V^{0}=f^{-\mathbf{n q}}\left(Z_{\kappa}^{1}\right) \mid 0=Y^{\mathbf{n q}+1} .
$$

Each puzzle piece $Z_{j}^{1}$ has $2^{m}$ univalent pullbacks under the $2^{m}$-covering $f^{\mathbf{q} m}: Y^{\mathbf{q} m} \rightarrow$ $Y^{0}, m=1, \ldots, \mathbf{n}-1$. We label these pullbacks (for all $j$ ) as $Z_{i}^{1+\mathbf{q} m}$. Then

$$
f^{\mathbf{q} m}(0) \in Z_{\boldsymbol{\kappa}_{m}}^{\mathbf{q}(\mathbf{n}-m)+1}, \quad m=1, \ldots, \mathbf{n},
$$

for some sequence $\bar{\kappa}=\left(\kappa_{1}, \ldots, \kappa_{n}=\kappa\right)$ called the escape route of the critical point. The escape route specifies the tip $t=t_{\kappa_{1} \ldots \kappa_{\mathbf{n}-1}}$ of $M$ such that $f_{t}$ satisfies (2.1) for $m<\mathbf{n}$, while $f^{\mathbf{q n}}=\alpha^{\prime}$.

There are $\mathbf{q}$ parameter rays landing at each tip $t$ of $M_{\mathbf{p} / \mathbf{q}}$. They chop off $\mathbf{q}-1$ decorations $\mathcal{L}_{\bar{\kappa}}$ (the components of $M \backslash\{t\}$ that do not intersect the main cardioid) from $M$. The limb $\mathcal{L}_{\bar{\kappa}}$ attached to $t$ is specified by the puzzle piece $Z_{\boldsymbol{\kappa}}^{1}$ containing $f^{\mathbf{q n}}(0)$. Note that there are only finitely many decorations with bounded $\mathbf{q}$ and $\mathbf{n}$.

Let $P=Y^{(\mathbf{n}-1) \mathbf{q}+1}$. The piece $P$ has $2^{\mathbf{n}}$ vertices each of which is a preimage of $\alpha$ of some depth $\mathbf{q} m$ with $m \leq \mathbf{n}$ (and it takes into account all preimages of $\alpha$ in $Y^{1}$ up to depth $\mathbf{q n}$ ).

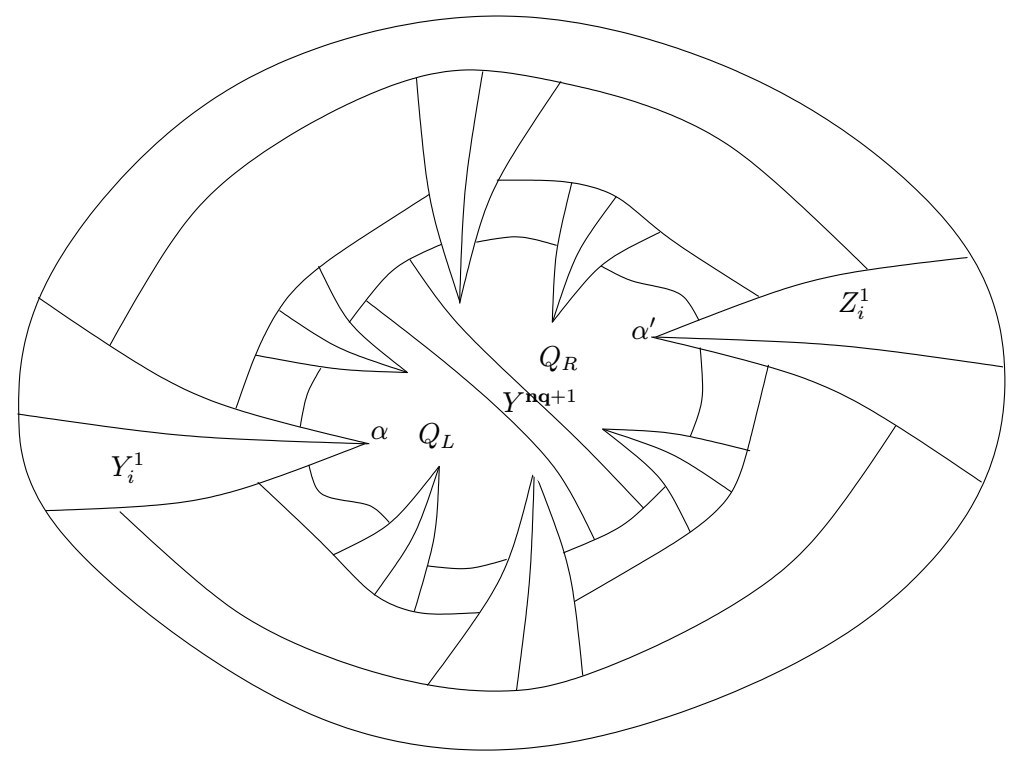

Figure 2.1. The top of the Yoccoz puzzle

Note that $f^{\mathbf{q}}(P) \supset P$ and the critical value $f^{\mathbf{q}}(0)$ does not belong to $P$. Hence $P$ has two univalent $f^{\mathbf{q}}$-pullbacks, $Q_{L}$ and $Q_{R}$ (of depth $\mathbf{q n}+1$ ), inside $P$. The puzzle piece $Q_{L}$ is attached to the fixed point $\alpha$ while $Q_{R}$ is attached to $\alpha^{\prime}$. Each of them shares two external rays with $V^{0}$. 
Lemma 2.1. - For any vertex $v$ of $P$, there exists a puzzle piece $Q^{v} \subset P$ of depth $(2 \mathbf{n}-1) \mathbf{q}+1$ attached to the boundary rays of $P$ landing at $v$, which is a univalent $f^{\mathbf{n q}}$-pullback of $P$. Moreover, these puzzle pieces are pairwise disjoint.

Proof. - Let $g=f^{\mathbf{q}} \mid Q_{L} \cup Q_{R}$. The domain of $g^{\mathbf{n}}$ consists of $2^{\mathbf{n}}$ components each of which is a univalent pullback of $P$. Each of these components contains a single $g^{\mathbf{n}}$-preimage $v$ of $\alpha$, and is attached to the pair of the boundary external rays of $P$ landing at $v$. This is the desired puzzle piece $Q^{v}$.

Given two vertices, $v$ and $w$, of $P$, we let $T_{P}^{v w}=K_{P} \backslash\left(Q^{v} \cup Q^{w}\right)$. Notice that $T^{v w}$ separates $v$ from $w$ in the sense that $v$ and $w$ belong to different components of $K_{P} \backslash T_{v w}$.

\subsection{Modified principal nest}

Given a critical puzzle piece $V$, let us consider the first return $f^{l} 0, l \in \mathbb{N}$, of the critical point to $V$ (whenever it exists). The corresponding pullback $W=f^{-l}(V) \mid 0$ of $V$ is called the central domain of the first return map to $V$, or briefly, the first child of $V$. Then, $W \subset V$ and the first return map $f^{l}: W \rightarrow V$ is a double branched covering.

Under the above circumstances, we also consider the first moment $k \in \mathbb{N}$ such that $f^{k l}(0) \notin W$ and then the first return $f^{t}(0) \in W, t>k l$, back to $W$ (whenever these moments are well defined). We call it the fine return to $W$, and the corresponding pullback $A=f^{-t}(W) \mid 0$ the fine child of $W$. The map $f^{t}: A \rightarrow W$ is a double branched covering. Note that if $f^{l}(0) \notin W$, the fine return coincides with the first return.

In [7] we have constructed a (Modified) Principle Nest of critical puzzle pieces

$$
E^{0} \ni E^{1} \ni E^{1} \ni \cdots \ni E^{\chi-1} \ni E^{\chi}
$$

and corresponding quadratic-like maps $g_{n}: E^{n} \rightarrow E^{n-1}$. Here for odd $n, E^{n}$ is the first child of $E^{n-1}$ and $g_{n}: E^{n} \rightarrow E^{n-1}$ is the corresponding first return map. For odd $n, E^{n}$ is the fine child of $E^{n-1}$ and $g_{n}: E^{n} \rightarrow E^{n-1}$ is the corresponding fine return map. We let $g \equiv g_{0}$.

If the map $f$ is renormalizable then the Principle Nest terminates at some odd level $\chi$. In this case, the last quadratic-like map $g_{\chi}: E^{\chi} \rightarrow E^{\chi-1}$ has a connected Julia set and represents the primitive renormalization $R f$ of $f$. The renormalization level $\chi$ is also called the height of the nest.

Primitively renormalizable parameter values assemble a maximal primitive copy $M^{\prime}$ of the Mandelbrot set $M$. This copy specifies the combinatorics of the renormalization in question. In particular, it determines the parameters $\mathbf{q}, \mathbf{n}$, the height $\chi$, and the renormalization period $p$.

In what follows we will assume that $f$ is primitively renormalizable. We let $\mathcal{K}=K(\mathcal{R} f)$ be the little (filled) Julia set of $f$, and we let $p$ be the renormalization period, i.e., $g_{\chi}=f^{p}$ so that $f^{p}(\mathcal{K})=\mathcal{K}$. We let $\mathcal{K}_{i}=f^{i}(\mathcal{K})$, where $i$ is taken $\bmod p$, which are also called "little Julia sets".

It is important to note that the maps $g_{n}$ admit analytic extensions $\tilde{E}^{n} \rightarrow \hat{E}^{n-1}$ such that $E^{n} \subset \tilde{E}^{n} \subset \hat{E}^{n} \subset E^{n-1}$ and for odd $n, \hat{E}^{n}=E^{n-1}[7]$, 2.4 . For $n=0$, we let $\hat{E}^{0}=\tilde{E}^{0}=$ $Y^{\mathbf{q n}}$. Then $f^{\mathbf{q n}}: \tilde{E}^{0} \rightarrow Y^{0}$ is a branched covering of degree $2^{\mathbf{n}}$.

The following observation will be used many times: 
Lemma 2.2 (Telescope). - Let $X_{k}$ be a sequence of topological disks, $k=0,1, \ldots, n$, and let $\phi_{k}: X_{k} \rightarrow \phi\left(X^{k}\right)$ be branched coverings of degree $d_{k}$ such that $\phi\left(X_{k}\right) \supset X_{k+1}$. Let $\Phi=\phi_{n-1} \circ \cdots \circ \phi_{0}$ (wherever it is defined), and let $P \subset X_{0}$ be a component of its domain of definition. Then $\Phi: P \rightarrow X_{n}$ is a branched covering of degree at most $d_{0} \cdots d_{n-1}$.

If the renormalization $R f$ is also renormalizable then $f$ is called twice renormalizable, and $R^{2} f$ stands for its second renormalization. Proceeding this way, we can define infinitely renormalizable maps $f$, and let $R: R^{n-1} f \mapsto R^{n} f$ be their $n$-fold renormalizations. The combinatorics of an infinitely renormalizable map is a sequence of little Mandelbrot copies $M^{(n)}$ that determine the combinatorics of the renormalizations $R^{n} f$. It determines the sequence of the parameters $\mathbf{q}_{n}, \mathbf{n}_{n}$, the heights $\chi_{n}$, and the periods $p_{n}$ of the corresponding renormalizations.

We say that an infinitely renormalizable $f$ satisfies the decoration condition if all the little copies $M^{(n)}$ belong to finitely many decorations $\mathcal{L}_{k}$. Equivalently, the parameters $\mathbf{q}_{n}$ and $\mathbf{n}_{n}$ are bounded.

\subsection{Geometric puzzle pieces}

In what follows we will deal with more general puzzle pieces.

Given a puzzle piece $Y_{i}^{m}$, let $Y_{i}^{m, l}$ stand for a Jordan disk bounded by the same external rays as $Y_{j}^{m}$ and arcs of equipotentials of level $l$ (so $Y_{i}^{m, m}=Y_{i}^{m}$ ). Such a disk will be called a puzzle piece of bidepth $(m, l)$.

A geometric puzzle piece of bidepth $(m, l)$ is a closed Jordan domain which is the union of several puzzle pieces of the same bidepth. As for ordinary pieces, a pullback of a geometric puzzle piece of bidepth $(m, l)$ under some iterate $f^{k}$ is a geometric puzzle piece of bidepth $(m+k, l+k)$. Note also that if $P$ and $P^{\prime}$ are geometric puzzle pieces with ${ }^{(2)}$ bidepth $P \geq$ bidepth $P^{\prime}$ and $K_{P} \subset K_{P^{\prime}}$ then $P \subset P^{\prime}$.

The family of geometric puzzle pieces of bidepth $(m, l)$ will be called $\mathcal{Y}^{m}(l)$. Given a geometric puzzle piece $Y \in \mathcal{Y}^{m}(l)$, we let $Y(k)$ be the puzzle piece bounded by the same external rays as $Y$ truncated by the equipotential of level $k$. (In particular, $Y(l)=Y$.)

Any puzzle piece $Y \in \mathcal{Y}^{m}(l)$ admits the following combinatorial representation. Let $\theta_{i}$ be the cyclically ordered angles of the external rays $\mathcal{R}_{i}$ that bound $Y$. Let us consider the straight rays $R_{i}$ in $\mathbb{C} \backslash \mathbb{D}$ of angles $\theta_{i}$ truncated by the circle $\mathbb{T}_{r}$ of radius $r=2^{2^{-l}}$. If two consecutive rays, $\mathcal{R}_{i}$ and $\mathcal{R}_{i+1}$, land at the same vertex of $Y$, let us connect $R_{i}$ to $R_{i+1}$ with a hyperbolic geodesic in $\mathbb{D}$. Otherwise $\mathcal{R}_{i}$ and $\mathcal{R}_{i+1}$ are connected with an equipotential arc. Then let us connect $R_{i}$ to $R_{i+1}$ with the appropriate arc of $\mathbb{T}_{r}$. We obtain a Jordan curve that bounds the combinatorial model $M_{Y}$ of $Y$.

The $\operatorname{arcs} \omega_{i}$ of $\mathbb{T} \cap M_{Y}$ correspond to the "external arcs" of the Julia piece $K_{Y}$. They have length $2 \pi \lambda$, where $\lambda$ is called the combinatorial length of the corresponding external arc of $K_{Y}$. In case $Y$ is a dynamical puzzle piece, all the external arcs of $Y$ have the same combinatorial length

$$
\frac{2^{k}}{\left(2^{\mathbf{q}}-1\right) 2^{m}}, \quad k \in\{0,1, \ldots, q-1\}
$$

(2) The inequality between bidepths is understood componentwise

$4^{\mathrm{e}}$ SÉRIE - TOME $41-2008-\mathrm{N}^{\mathrm{o}} 1$ 
where the choice of $k$ depends on the puzzle piece $f^{m}(Y)$ of depth 0 . (For instance, $k=0$ when $f^{m}(Y)$ contains the critical value $f(0)$, while $k=q-1$ when $f^{m}(Y)$ contains the critical point 0 .)

It follows that for a geometric puzzle piece $Y$ of depth $m$, the combinatorial length of its external arcs is at least $2^{-(\mathbf{q}+m)}$.

Let us now consider a geometric puzzle piece $Z^{0}=-Y^{0}$ of bidepth $(1,0)$.

Lemma 2.3. - Let $z \in K(f), f^{\mathbf{q n}} z \in Z^{0}$ and let $P=f^{-\mathbf{q n}}\left(Z^{0}\right) \mid z$. Then $P \Subset \operatorname{int} Y^{0}$ or $P \Subset \operatorname{int} Z^{0}$.

Proof. $-P$ is a geometric puzzle piece of bidepth $(\mathbf{n q}+1, \mathbf{n q})$. But $E^{0}=Y^{\mathbf{n q}+1}$ is a puzzle piece of depth $\mathbf{n q}+1$ such that $f^{\mathbf{q n}}\left(E^{0}\right)=Z_{\boldsymbol{\kappa}}$, where int $Z_{\boldsymbol{\kappa}} \cap Z^{0}=\varnothing$. It follows that $P \cap \operatorname{int} E^{0}=\varnothing$. But $K(f) \backslash$ int $E^{0}$ consists of two 0 -symmetric connected components $X_{L} \supset L \cap K(f)$ and $X_{R} \supset R \cap K(f)$. We conclude that $K_{P}$ is contained in one of these components, and hence it is contained in one of the sets $K_{Z^{0}}$ or $K_{Y^{0}}$. As

$$
\text { bidepth } P \geq(1,0)=\operatorname{bidepth} Z^{0} \geq(0,0)=\operatorname{bidepth} Y^{0},
$$

$P$ is contained in one of the puzzle pieces $Z^{0}$ or $Y^{0}$.

\subsection{Many happy returns}

Here we will summarize the combinatorial construction of [7], §1.9, that will lead to the moduli improvement in the case of high type.

Fix an arbitrary $m$, let $N$ be the smallest even integer which is bigger than $\log _{2} m+5$, and take any odd level $n \geq N$. Then there exist $m / 2$ returns $\Lambda_{k}=g^{l_{k}}\left(E^{n}\right)$ of the domain $E^{n}$ to $E^{n-N}$ with the following properties. For any domain $\Lambda_{k}$, the map $\Psi_{k}=g^{l_{k}}: E^{n} \rightarrow \Lambda_{k}$ admits a holomorphic extension to a branched covering

$$
\Psi_{k}:\left(\Upsilon_{k}, \Delta_{k}, E^{n}\right) \rightarrow\left(E^{n-N-1}, \Lambda_{k}^{\prime}, \Lambda_{k}\right)
$$

such that:

(P1) $\operatorname{deg}\left(\Psi_{k}: \Upsilon_{k} \rightarrow E^{n-N-1}\right) \leq 2^{N+m}$;

(P2) $\operatorname{deg}\left(\Psi_{k}: \Delta_{k} \rightarrow \Lambda_{k}^{\prime}\right) \leq d^{5}$;

(P3) $\Upsilon_{k} \subset E^{n-1}$;

(P4) there is a level $i \in[n-5, n-1]$ such that each pair of disks $\left(\Lambda_{k}^{\prime}, \Lambda_{k}\right)$ is mapped univalently onto $\left(\hat{E}^{i}, E^{i}\right)$ under some iterate $f^{t}, t=t(k)$;

(P5) the buffers $\Lambda_{k}^{\prime} \Subset E^{n-N}$ are pairwise disjoint.

\section{Pseudo-quadratic-like maps and pseudo-puzzle}

\subsection{Pseudo-quadratic-like maps}

For a more general and detailed discussion of $\psi$-ql maps, see [6].

Suppose that $\mathbf{U}^{\prime}, \mathbf{U}$ are disks, and $i: \mathbf{U}^{\prime} \rightarrow \mathbf{U}$ is a holomorphic immersion, and $f: \mathbf{U}^{\prime} \rightarrow \mathbf{U}$ is a degree $d$ holomorphic branched cover. Suppose further that there exist full continua $K \Subset \mathbf{U}$ and $K^{\prime} \Subset \mathbf{U}^{\prime}$ such that $K^{\prime}=i^{-1}(K)=f^{-1}(K)$. Then we say that $F=(i, f): \mathbf{U}^{\prime} \rightarrow \mathbf{U}$ is a $\psi$-quadratic-like $(\psi$-ql) map with filled Julia set $K$. 
Lemma $3.1([6])$. - Let $F=(i, f): \mathbf{U}^{\prime} \rightarrow \mathbf{U}$ be a $\psi$-ql map of degree $d$ with filled Julia set $K$. Then $i$ is an embedding in a neighborhood of $K^{\prime} \equiv f^{-1}(K)$, and the map $g \equiv f \circ i^{-1}: U^{\prime} \rightarrow U$ near $K$ is quadratic-like.

Moreover, the domains $U$ and $U^{\prime}$ can be selected in such a way that $\bmod \left(U \backslash i\left(U^{\prime}\right)\right) \geq$ $\mu(\bmod (\mathbf{U} \backslash K))>0$.

There is a natural $\psi$-ql map $\mathbf{U}^{n} \rightarrow \mathbf{U}^{n-1}$, the "restriction" of $(i, f)$ to $\mathbf{U}^{n}$. Somewhat loosely, we will use the same notation $F=(i, f)$ for this restriction.

Let us normalize the $\psi$-quadratic-like maps under consideration so that diam $K^{\prime}=$ $\operatorname{diam} K=1$, both $K$ and $K^{\prime}$ contain 0 and 1, 0 is the critical point of $f$, and $i(0)=0$. Let us endow the space of $\psi$-quadratic-like maps (considered up to independent rescalings in the domain and the range) with the Carathéodory topology. In this topology, a sequence of normalized maps $\left(i_{n}, f_{n}\right): \mathbf{U}_{n}^{\prime} \rightarrow \mathbf{U}_{n}$ converges to $(i, f): \mathbf{U}^{\prime} \rightarrow \mathbf{U}$ if the pointed domains $\left(\mathbf{U}_{n}^{\prime}, 0\right)$ and $\left(\mathbf{U}_{n}, 0\right)$ converge to $\mathbf{U}^{\prime}$ and $\mathbf{U}$ respectively, and the maps $i_{n}, f_{n}$ converge respectively to $i, f$, uniformly on compact subsets of $\mathbf{U}^{\prime}$.

Lemma 3.2 (compare [10]). - Let $\mu>0$. Then the space of $\psi$-ql maps $F=(i, f)$ : $\mathbf{U}^{\prime} \rightarrow \mathbf{U}$ such that the Julia set $K$ is connected and $\bmod (\mathbf{U} \backslash K) \geq \mu$ is compact.

Proof. - Let $X_{n}=i_{n}^{-1}\{0,1\}, Y_{n}=f_{n}^{-1}\{0,1\}$. Note that both sets consist of at most 3 points and are contained in $\mathbb{D}$.

Then we can select a subsequence of domains $\mathbf{U}_{n}^{\prime}, \mathbf{U}_{n}$ Carathéodory converging to some domains $\mathbf{U}^{\prime}, \mathbf{U}$, while the sets $X_{n}$ and $Y_{n}$ converge in the Hausdorff metric to some sets $X \subset$ $\mathbf{U}^{\prime}$ and $Y \subset \mathbf{U}^{\prime}$ that consist of at most three points and are contained in $\mathbb{D}$ (we will keep the same notation for the subsequence). Since the maps $i_{n} \mid \mathbf{U}_{n}^{\prime} \backslash X_{n}$ and $f_{n} \mid \mathbf{U}_{n}^{\prime} \backslash Y_{n}$ do not assume values 0 and 1 , they form normal families on $\mathbf{U}^{\prime} \backslash X, \mathbf{U}^{\prime} \backslash Y$. Since these families are bounded on the sets $K_{n}$, they are uniformly bounded on compact sets of $\mathbf{U}^{\prime} \backslash(X \cup Y)$. By the Maximum Principle, they are normal on the whole domain $\mathbf{U}^{\prime}$.

Let $i$ and $f$ be some limit functions of the sequences $i_{n}$ and $f_{n}$. These functions are nonconstant since they assume values 0 and 1 . Then $i$ is an immersion as a non-constant limit of immersions. Also, $f: \mathbf{U}^{\prime} \rightarrow \mathbf{U}$ is a branched covering of degree at most 2. Moreover, $K^{\prime} \Subset \mathbf{U}$ since $\bmod \left(\mathbf{U}^{\prime} \backslash K^{\prime}\right) \geq \mu / 2$. Hence $0 \in \mathbf{U}^{\prime}$, and it is a critical point of $f$. It follows that $\operatorname{deg} f=2$, and we are done.

\subsection{Pseudo-puzzle}

3.2.1. Definitions. - Let $(i, f): \mathbf{U}^{\prime} \rightarrow \mathbf{U}$ be a $\psi$-ql map. By Lemma 3.1, it admits a quadratic-like restriction $U^{\prime} \rightarrow U$ to a neighborhood of its (filled) Julia set $K=K_{\mathbf{U}}$. Here $U^{\prime}$ is embedded to $U$, so we can identify $U^{\prime}$ with $i\left(U^{\prime}\right)$ and $f: U^{\prime} \rightarrow U$ with $f \circ i^{-1}$.

Assume that $K$ is connected and both fixed points of $f$ are repelling. Then we can cut $U$ by external rays landing at the $\alpha$-fixed point and consider the corresponding Yoccoz puzzle.

Given a (geometric) puzzle piece $Y$ of depth $m$, recall that $K_{Y}$ stands for $Y \cap K(f)$ and $S_{Y}=\operatorname{cl}\left(K(f) \backslash K_{Y}\right)$. Let $\mathcal{C}$ stand for the space of paths $\delta:[0,1] \rightarrow \mathbf{U}^{m} \backslash S_{Y}$ such that:

- $\delta(0) \in Y$, 
- if $\delta(t) \in K_{Y}$, then the restriction $\delta \mid[0, t]$ is homotopic relatively to endpoints to a path contained in $Y{ }^{(3)}$

Let $\mathbf{Y}$ be the space of paths $\delta \in \mathcal{C}$ modulo homotopy through $\mathcal{C}$ with $\delta(1)$ fixed. Define the projection $\pi_{Y}: \mathbf{Y} \rightarrow \mathbf{U}^{m}$ by $[\delta] \mapsto \delta(1)$. One can see that $\mathbf{Y}$ is a Riemann surface, and $\pi_{Y}$ is an immersion such that $Y$ lifts to a disk $\hat{Y} \subset \mathbf{Y}$ which is homeomorphically projected onto $Y$. Thus, we can identify $\hat{Y}$ with $Y$; in particular, $K_{Y}$ is embedded into $\mathbf{Y}$.

The Riemann surface $\mathbf{Y}$ will be called the pseudo-piece (" $\psi$-piece") associated with $Y$.

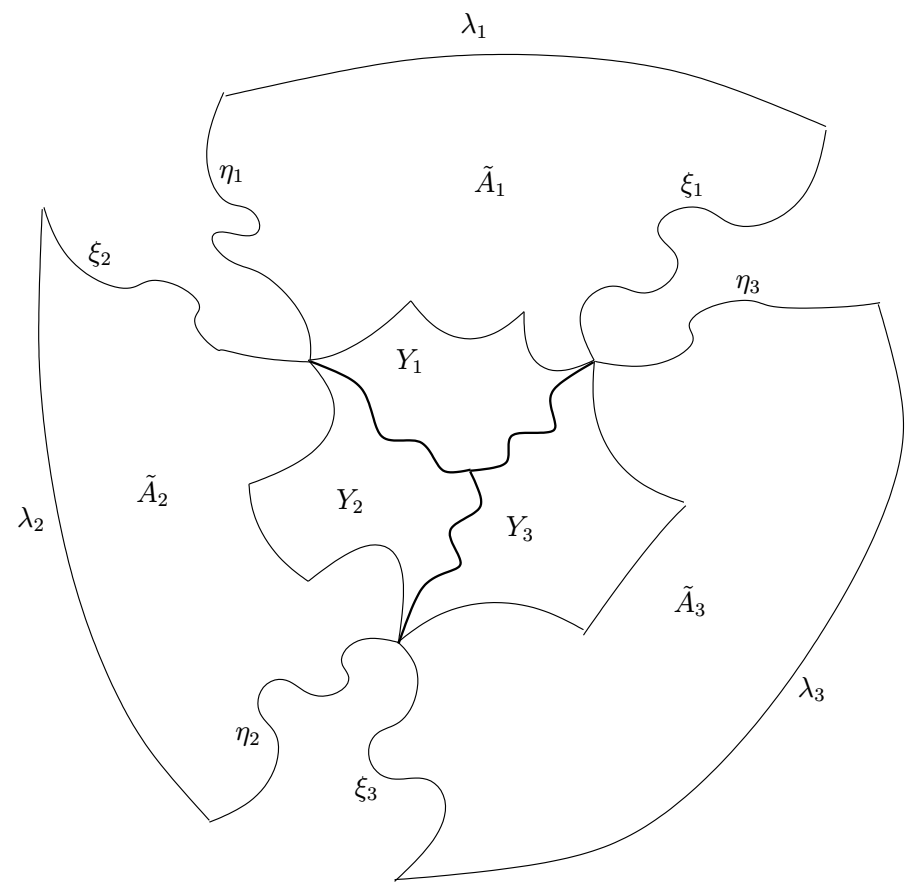

Figure 3.1. The pseudo-puzzle piece

The $\psi$-pieces can also be defined in a different way (see Figure 3.1). Let us consider the topological annulus $A=\mathbf{U}^{m} \backslash K(f)$ and its universal covering $\hat{A}$. Let $Y_{i}$ be the components of $Y \backslash K_{Y}$. There are finitely many of them, and each $Y_{i}$ is simply connected. Hence they can be embedded into $\hat{A}$. Select such an embedding $e_{i}: Y_{i} \rightarrow \hat{A}_{i}$ where $\hat{A}_{i}$ stands for a copy of $\hat{A}$. Then the $\psi$-piece is obtained by gluing the $A_{i}$ to $Y$ by means of $e_{i}$, i.e., $\mathbf{Y}=Y \sqcup_{e_{i}} \hat{A}_{i}$.

Lemma 3.3. - The above two definitions of $\psi$-pieces are equivalent.

Proof. - Let $\mathbf{Y}$ be a $\psi$-piece according to the first definition. The puzzle piece $Y$ is embedded into $\mathbf{Y}$ by associating to a point $y \in Y$ the constant path $\delta(t) \equiv y$.

(3) This condition can be replaced with a more restrictive one: After the first exit from $Y$, the path never intersects the Julia set $K(f)$ (though it is allowed to return back to $Y$ ). 
Let us realize the universal covering $\hat{A}_{i} \rightarrow A$ as the space of paths in $A$ that begin in $Y_{i}$ relatively to homotopy through such paths fixing the terminal endpoint. (This realization is legitimate since $Y_{i}$ is simply connected.) This provides us with an embedding $\phi_{i}: \hat{A}_{i} \rightarrow \mathbf{Y}$.

The embeddings $\phi_{i}$ have disjoint images. Indeed, all points of $\partial Y \cap K(f)$ are dividing and thus belong to $S_{Y}$. Hence, if we take two paths $\delta_{1}:[0,1] \rightarrow \mathbf{U}^{m} \backslash K(F)$ and $\delta_{2}:[0,1] \rightarrow$ $\mathbf{U}^{m} \backslash K(f)$ as above representing points in $\hat{A}_{i}$ and $\hat{A}_{j}(i \neq j)$ with a common endpoint, then they "surround" some piece of $S_{Y}$, and hence represent different points in $\mathbf{Y}$.

Moreover, the image $\phi_{i}\left(\hat{A}_{i}\right)$ overlaps with $Y$ by $Y_{i}$. Hence we obtain an embedding of $Y \sqcup_{e_{i}} \hat{A}_{i}$ into $\mathbf{Y}$.

Let us show that this embedding is surjective. Take a path $\delta \in \mathcal{C}$ representing some point of $\mathbf{Y}$, and let $\tau \in[0,1]$ be the last parameter for which $\delta(\tau) \in K_{Y}$. Since the path $\delta:[0, \tau] \rightarrow$ $\mathbf{U}^{m}$ is trivial (i.e., it can be pulled to $Y$ in $\mathbf{U}^{m} \backslash S_{Y}$ relatively to endpoints), the restriction $\delta:[\tau, 1] \rightarrow \mathbf{U}^{m} \backslash S_{Y}$ (appropriately reparametrized) represents the same point in $\mathbf{Y}$ as the original path. Moreover, if $\tau \neq 1$, we can replace it with an equivalent path $\delta:[\tau+\varepsilon, 1] \rightarrow$ $\mathbf{U}^{m} \backslash S_{Y}$ which is disjoint from the Julia set $K(f)$. As the latter path represents a point in some $\hat{A}_{i}$, we are done.

\subsubsection{Naturality}

Lemma 3.4. - (i) Consider two puzzle pieces $Y$ and $Z$ such that the map $f: Y \rightarrow Z$ is a branched covering of degree $k$ (where $k=1$ or $k=2$ depending on whether $Y$ is off-critical or not). Then there exists an induced map $\mathbf{f}: \mathbf{Y} \rightarrow \mathbf{Z}$ which is a branched covering of the same degree $k$.

(ii) Given two puzzle pieces $Y \subset Z$, the inclusion $i: Y \rightarrow Z$ extends to an immersion $\mathbf{i}: \mathbf{Y} \rightarrow \mathbf{Z}$.

Proof. - Both properties follow easily from either definition of the $\psi$-pieces. Let us, for instance, use the second definition.

(i) Let depth $Y=m$, depth $Z=m-1$. Let us consider the degree $k$ branched covering

$$
f:\left(\mathbf{U}^{m}, Y, K_{Y}\right) \rightarrow\left(\mathbf{U}^{m-1}, Z, K_{Z}\right) .
$$

The components $Y_{i}$ of $Y \backslash K_{Y}$ are univalently mapped onto components $Z_{j(i)}$ of $Z \backslash K_{Z}$, where the map $j=j(i)$ is $k$-to-1. This map extends to an isomorphism map $\hat{A}_{i} \rightarrow \hat{B}_{j}$ of the corresponding universal coverings, which glue together into a branched covering $\mathbf{Y} \rightarrow \mathbf{Z}$ of degree $k$.

(ii) Let $\operatorname{depth} Y=m$, depth $Z=n$. Let us consider the immersion

$$
i:\left(\mathbf{U}^{m}, Y, K_{Y}\right) \rightarrow\left(\mathbf{U}^{n}, Z, K_{Z}\right) .
$$

The components $Y_{i}$ are embedded by $i$ into some components $Z_{j(i)}$, where the map $j=j(i)$ is surjective but not necessarily injective. These embeddings extend to immersions $\hat{A}_{i} \rightarrow \hat{B}_{j}$ that glue together into an immersion $\mathbf{Y} \rightarrow \mathbf{Z}$.

$4^{\mathrm{e}}$ SÉRIE - TOME $41-2008-\mathrm{N}^{\mathrm{o}} 1$ 
3.2.3. Moduli. - Given two puzzle pieces $Z \Subset Y$, we let

$$
\bmod (Y, Z)=\bmod \left(\mathbf{Y} \backslash K_{Z}\right) .
$$

Lemma 3.4 implies:

Lemma 3.5. - (i) Consider two pairs of puzzle pieces $\left(Y^{\prime}, Y\right)$ and $\left(Z^{\prime}, Z\right)$ such that the map $f:\left(Y^{\prime}, Y\right) \rightarrow\left(Z^{\prime}, Z\right)$ is a branched covering of degree $k$ (on both domains). Then

$$
\bmod \left(Z^{\prime}, Z\right)=k \bmod \left(Y^{\prime}, Y\right) .
$$

(ii) Given a nest of three puzzle pieces $W \subset Z \subset Y$, we have

$$
\bmod (Z, W) \leq \bmod (Y, W) .
$$

3.2.4. Boundary of pseudo-puzzle pieces. - Let us mention in conclusion that the ideal boundary of a pseudo-puzzle $\mathbf{Y}$ is tiled by (finitely many) $\operatorname{arcs} \lambda_{i} \subset \partial \hat{A}_{i}$ that cover the ideal boundary of $\mathbf{U}^{m}$ (where $m=\operatorname{depth} Y$ ) and $\operatorname{arcs} \xi_{i}, \eta_{i} \subset \partial \hat{A}_{i}$ mapped onto the Julia set $J(f)$ (see Figure 3.1). The arc $\lambda_{i}$ meets each $\xi_{i}, \eta_{i}$ at a single boundary point corresponding to a path $\delta:[0,1) \mapsto A$ that wraps around $K(f)$ infinitely many times, while $\eta_{i}$ meets $\xi_{i+1}$ at a vertex $v_{i} \in Y \cap K(f)$. We say that the arcs $\lambda_{i}$ form the outer boundary (or "O-boundary") $\partial_{O} \mathbf{Y}$ of the puzzle piece $\mathbf{Y}$, while the $\operatorname{arcs} \xi_{i}$ and $\eta_{i}$ form its $J$-boundary $\partial_{J} \mathbf{Y}$. Given a vertex $v=v_{i}$ of a puzzle piece $Y$, let $\partial^{v} \mathbf{Y}=\eta_{i} \cup \xi_{i+1}$ stand for the part of the $J$-boundary of $\mathbf{Y}$ attached to $v$.

Note that the immersion constructed in Lemma 3.4 extends continuously to the boundary of the puzzle piece $\mathbf{Y}$. (However, $\mathbf{i}(\partial \mathbf{Y})$ is not contained in $\partial \mathbf{Z}$, unless $Z=Y$.) In what follows we will assume this extension without further comment.

A multicurve in some space $X$ is a continuous map $\gamma: \cup_{k=1}^{l}\left[s_{k}, t_{k}\right] \rightarrow X$ parametrized by a finite union of disjoint intervals $\left[s_{k}, t_{k}\right] \subset \mathbb{R}$. ${ }^{(4)}$ Note that multicurves are ordered. A multicurve in a puzzle piece $\mathbf{Y}$ is called horizontal if

$$
\gamma\left(s_{1}\right) \in \partial^{v_{0}} \mathbf{Y}, \quad \gamma\left(t_{k}\right), \gamma\left(s_{k+1}\right) \in \partial^{v_{k}} \mathbf{Y}, k=1, \ldots, l-1, \quad \gamma\left(t_{l}\right) \in \partial^{v_{l}} \mathbf{Y}
$$

for some vertices $v_{k}$ of $\mathbf{Y}, k=0, \ldots, l$. We say that such a multicurve "connects" $\partial^{v_{0}} \mathbf{Y}$ to $\partial^{v_{l}} \mathbf{Y}$. The following statement motivates introduction of multicurves:

Lemma 3.6. - Let $v$ and $w$ be two vertices of a geometric puzzle piece $Y \in \mathcal{Y}^{m}(l)$. Then any curve $\gamma$ in $\mathbf{U}^{l}$ connecting $S_{Y}^{v}$ to $S_{Y}^{w}$ contains a multicurve $\gamma^{\prime}$ that lifts to a multicurve $\gamma^{*}$ in $\mathbf{Y}$ connecting $\partial^{v} \mathbf{Y}$ to $\partial^{w} \mathbf{Y}$.

Given two vertices $v$ and $w$ of $Y$, let $\mathcal{G}_{Y}(v, w)$ stand for the family of horizontal multicurves in $\mathbf{Y}$ connecting $\partial^{v} \mathbf{Y}$ to $\partial^{w} \mathbf{Y}$. Finally, let

$$
\mathbf{d}_{Y}(v, w)=\mathcal{L}\left(\mathcal{G}_{Y}^{v w}\right)
$$

stand for the extremal distance between the corresponding parts of $J$-boundary of $\mathbf{Y}$.

Lemma 3.7. - If $f \mid Y$ is univalent, then $\mathbf{d}_{Y}(v, w)=\mathbf{d}_{f(Y)}(f v, f w)$.

(4) We allow that the boundary points of a multicurve in a pseudo puzzle $\mathbf{Y}$ belong to $\partial_{J} \mathbf{Y}$. 


\section{Quasi-Additivity Law and Covering Lemma}

Let us now formulate two analytic results which will play a crucial role in what follows. The first one appears in $\S 2.10 .3$ of [8]:

Quasi-Additivity Law. - Fix some $\eta \in(0,1)$. Let $\mathbf{V}$ be a topological disk, let $K_{i} \Subset$ $\mathbf{V}, i=1, \ldots, m$, be pairwise disjoint full compact continua, and let $\phi_{i}: \mathbb{A}\left(1, r_{i}\right) \rightarrow \mathbf{V} \backslash \cup K_{j}$ be holomorphic annuli such that each $\phi_{i}$ is an embedding of some proper collar of $\mathbb{T}$ to a proper collar of $\partial K_{i}$. Then there exists a $\delta_{0}>0$ (depending on $\eta$ and $m$ ) such that: if for some $\delta \in$ $\left(0, \delta_{0}\right), \bmod \left(\mathbf{V}, K_{i}\right)<\delta$ while $\log r_{i}>2 \pi \eta \delta$ for all $i$, then

$$
\bmod \left(\mathbf{V}, \cup K_{i}\right)<\frac{2 \eta^{-1} \delta}{m} .
$$

The next result appears in $\S 3.1 .5$ of [8]:

Covering Lemma. - Fix some $\eta \in(0,1)$. Let us consider two topological disks $\mathbf{U}$ and $\mathbf{V}$, two full continua $A^{\prime} \subset \mathbf{U}$ and $B^{\prime} \subset \mathbf{V}$, and two compact subsets, $A \subset A^{\prime}$ and $B \subset B^{\prime}$, of topological type bounded by $T$. $^{(5)}$

Let $f: \mathbf{U} \rightarrow \mathbf{V}$ be a branched covering of degree $D$ such that $A^{\prime}$ is a component of $f^{-1}\left(B^{\prime}\right)$, and $A$ is the union of some components of $f^{-1}(B)$. Let $d=\operatorname{deg}\left(f: A^{\prime} \rightarrow B^{\prime}\right)$.

Let $B^{\prime}$ be also embedded into another topological disk $\mathbf{B}^{\prime}$. Assume $\mathbf{B}^{\prime}$ is immersed into $\mathbf{V}$ by a map $i$ in such a way that $i \mid B^{\prime}=\mathrm{id}, i^{-1}\left(B^{\prime}\right)=B^{\prime}$, and $i\left(\mathbf{B}^{\prime}\right) \backslash B^{\prime}$ does not contain the critical values of $f$. Under the following "Collar Assumption":

$$
\begin{gathered}
\bmod \left(\mathbf{B}^{\prime}, B\right)>\eta \bmod (\mathbf{U}, A), \\
\text { if } \bmod (\mathbf{U}, A)<\varepsilon(\eta, T, D) \text { then } \bmod (\mathbf{V}, B)<2 \eta^{-1} d^{2} \bmod (\mathbf{U}, A) .
\end{gathered}
$$

\section{Improving the moduli}

In this section $C=2$ will stand for the constant in the Quasi-Additivity Law and the Covering Lemma.

\subsection{High type}

Let us begin with a simple estimate that compares moduli on consecutive odd levels of the Principal Nest:

Lemma 5.1. - For any odd n, we have:

$$
\bmod \left(E^{n-3}, E^{n-2}\right) \leq 4 \bmod \left(E^{n-1}, E^{n}\right)
$$

and

$$
\bmod \left(Y^{0}, R\right) \leq 2^{\mathbf{n}+1} \bmod \left(E^{0}, E^{1}\right)
$$

(5) In applications, $A$ and $B$ will be full continua, so $T=1$.

$4^{\text {e }}$ SÉRIE - TOME $41-2008-\mathrm{N}^{\mathrm{o}} 1$ 
Proof. - By Lemma 3.5,

$$
\bmod \left(\hat{E}^{n-1}, E^{n-1}\right)=2 \bmod \left(\tilde{E}^{n}, E^{n}\right) \leq 2 \bmod \left(E^{n-1}, E^{n}\right)
$$

and

$$
\begin{gathered}
\bmod \left(\hat{E}^{n-1}, E^{n-1}\right) \geq \bmod \left(\tilde{E}^{n-1}, E^{n-1}\right) \\
=\frac{1}{2} \bmod \left(\hat{E}^{n-2}, E^{n-2}\right)=\frac{1}{2} \bmod \left(E^{n-3}, E^{n-2}\right),
\end{gathered}
$$

and the first estimate follows.

The second estimate is similar. The puzzle piece $E^{1} \equiv Y^{m}$ is mapped with degree 2 onto $E^{0}$, and this map admits degree 2 extension $\tilde{E}^{1} \rightarrow Y^{q \mathbf{n}} \equiv \hat{E}^{0}$, where $\tilde{E}^{1}=Y^{m-1}$. Then $E^{0}$ is mapped onto $Z_{\kappa}^{1}$ by degree 2 map $f^{q \mathbf{n}}$. This map admits degree $2^{\mathbf{n}}$ extension $Y^{\mathbf{q n}} \rightarrow Y^{0}$. It follows that

$$
\begin{gathered}
\bmod \left(Y^{0}, R\right) \leq \bmod \left(Y^{0}, Z_{\boldsymbol{\kappa}}^{1}\right) \\
=2^{\mathbf{n}+1} \bmod \left(Y^{m-1}, E^{1}\right) \leq 2^{\mathbf{n}+1} \bmod \left(E^{0}, E^{1}\right) .
\end{gathered}
$$

The following lemma tells us that if some principal modulus is very small, then it should be even smaller on some preceding level of the Principal Nest:

Lemma 5.2. - There exist absolute $N \in \mathbb{N}$ and $\varepsilon>0$ such that: If on some odd level $n \geq N, \bmod \left(E^{n-1}, E^{n}\right)<\varepsilon$, then on some previous odd level $n-s \in[n-N, n-1]$ we have:

$$
\bmod \left(E^{n-s-1}, E^{n-s}\right)<\frac{1}{2} \bmod \left(E^{n-1}, E^{n}\right) .
$$

Proof. - Let us fix some integer $m>C^{3} 2^{28}$. Let $N$ be the smallest odd integer that is bigger than $\log _{2} m+5$. Take any odd level $n \geq N$. For each $k$, let us consider the associated 3-domain branched covering $\Psi_{k}$ (2.3)

$$
\Psi_{k}:\left(\Upsilon_{k}, \Delta_{k}, E^{n}\right) \rightarrow\left(E^{n-N}, \Lambda_{k}^{\prime}, \Lambda_{k}\right) .
$$

Let us consider two cases:

Case 1. Assume that for some domain $\Lambda_{k}$,

$$
\bmod \left(\Lambda_{k}^{\prime}, \Lambda_{k}\right) \leq \frac{1}{4} \bmod \left(E^{n-1}, E^{n}\right) .
$$

By Property $(\mathrm{P} 4)$ and Lemma 3.5, $\bmod \left(\Lambda_{k}^{\prime}, \Lambda_{k}\right)=\bmod \left(\hat{E}^{i}, E^{i}\right)$. If $i$ is odd then $\hat{E}^{i}=$ $E^{i-1}$, and we obtain the desired estimate with $s=n-i \in[1,5]$ :

$$
\bmod \left(E^{n-1}, E^{n}\right) \geq 4 \bmod \left(E^{i-1}, E^{i}\right) .
$$

If $i$ is even, then

$$
\bmod \left(\hat{E}^{i}, E^{i}\right) \geq \bmod \left(\tilde{E}^{i}, E^{i}\right)=\frac{1}{2} \bmod \left(\hat{E}^{i-1}, E^{i-1}\right)=\frac{1}{2} \bmod \left(E^{i-2}, E^{i-1}\right),
$$

and we conclude that

$$
\bmod \left(E^{n-1}, E^{n}\right) \geq 2 \bmod \left(E^{i-2}, E^{i-1}\right)
$$


Case 2. Assume that for all $\Lambda_{k}$,

$$
\bmod \left(\Lambda_{k}^{\prime}, \Lambda_{k}\right) \geq \frac{1}{4} \bmod \left(E^{n-1}, E^{n}\right) \geq \frac{1}{4} \bmod \left(\Upsilon_{k}, E^{n}\right)
$$

(where the second estimate follows from the inclusion $\Upsilon_{k} \subset E^{n-1}$ ). By Lemma 3.4, there exist a natural covering map

$$
\boldsymbol{\Psi}_{k}:\left(\boldsymbol{\Upsilon}_{k}, K_{\Delta_{k}}, K_{E^{n}}\right) \rightarrow\left(\mathbf{E}^{n-N-1}, K_{\Lambda_{k}^{\prime}}, K_{\Lambda_{k}}\right),
$$

and a natural immersion $i: \boldsymbol{\Lambda}_{k}^{\prime} \rightarrow \mathbf{E}^{n-N}$. Note that $i\left(\boldsymbol{\Lambda}_{k}^{\prime}\right) \backslash K_{\Lambda_{k}^{\prime}}$ does not contain the critical values of $\boldsymbol{\Psi}_{k}$, since the latter are contained in the Julia set $K(f)$. Moreover, estimate (5.2) provides us with the Collar Assumption that allows us to apply the Covering Lemma to the map $\boldsymbol{\Psi}_{k}$. If $\varepsilon$ is sufficiently small, it yields:

$$
\bmod \left(E^{n-N-1}, \Lambda_{k}\right) \leq C 2^{12} \bmod \left(\Upsilon_{k}, E^{n}\right) \leq C 2^{12} \bmod \left(E^{n-1}, E^{n}\right) .
$$

Estimates (5.2) and (5.3) show that the Quasi-Additivity Law is applicable to the family of continua $K_{\Lambda_{k}}$ in $\mathbf{E}^{n-N-1}$ with $\eta^{-1}=C 2^{14}$. Since there are at least $m / 2$ domains $\Lambda_{k} \subset$ $\Lambda_{k}^{\prime} \subset E^{n-N}$, it implies:

$$
\bmod \left(E^{n-N-1}, E^{n-N}\right) \leq \frac{C^{3} 2^{27} \bmod \left(E^{n-1}, E^{n}\right)}{m}<\frac{1}{2} \bmod \left(E^{n-1}, E^{n}\right),
$$

and we are done.

Lemma 5.3. - There exist absolute constants $C>0, \rho \in(0,1)$ and $\varepsilon>0$ such that if for some odd $n, \bmod \left(E^{n-1}, E^{n}\right)<\varepsilon$, then

$$
\bmod \left(E^{0}, E^{1}\right)<C \rho^{n} \bmod \left(E^{n-1}, E^{n}\right)
$$

and

$$
\bmod \left(Y^{0}, R\right) \leq C 2^{\mathbf{n}} \rho^{n} \bmod \left(E^{n-1}, E^{n}\right)
$$

Proof. - By Lemma 5.2, there exists an odd level $l<N$ such that

$$
\bmod \left(E^{l-1}, E^{l}\right) \leq\left(\frac{1}{2}\right)^{[n / N]} \bmod \left(E^{n-1}, E^{n}\right)
$$

which together with Lemma 5.1 implies the desired estimates.

\subsection{Frequent $R$-returns}

Let us consider the map

$$
f^{l}=f^{q \mathbf{n}+1} \circ g_{1} \circ \cdots \circ g_{\chi-1}: E^{\chi-1} \rightarrow Y^{0}
$$

and the trajectory $\mathcal{O}=\left\{\mathcal{K}_{i}\right\}_{i=l}^{l+p-1}$ of the little Julia set $\mathcal{K}$. Let $i_{1}, i_{2}, \ldots$ be the moments in $\mathcal{O}$ for which $\mathcal{K}_{i} \subset R$.

Lemma 5.4. - Let $\rho>0, \bar{\chi} \in \mathbb{N}$. Take some integer $m \geq C^{3} 2^{30} / \rho$, and let $\underline{p}=m^{2} \mathbf{q n}$. Assume that the little Julia set frequently visits $R$ :

$$
i_{k+1}-i_{k} \leq m \mathbf{q n}, \quad k=1,2, \ldots, m .
$$


If $\chi \leq \bar{\chi}$ while $p \geq \underline{p}$, then

$$
\bmod \left(Y^{0}, R\right) \leq \rho \bmod \left(\mathbf{E}^{\chi-1}, \mathcal{K}\right),
$$

provided $\bmod \left(\mathbf{E}^{\chi-1}, \mathcal{K}\right)<\varepsilon(\mathbf{n} ; \bar{\chi}, \rho)$.

Proof. - The map (5.4) has degree $2^{\chi} \leq 2^{\bar{\chi}}$. By Lemma 2.9 of [7], $\operatorname{deg}\left(f^{l} \mid E^{\chi}\right) \leq 32$, and hence $l \leq 5 p$.

By (5.5), $i_{m}-i_{1}<m^{2} \mathbf{q n} \leq p$, so the moments $i_{k}$ are pairwise non-congruent $\bmod p$. Hence the little Julia sets $\mathcal{K}_{i_{1}}, \ldots, \mathcal{K}_{i_{m}}$ are all distinct.

Since $\mathcal{O}$ has length $p$, there is only one critical Julia set in $\mathcal{O}$. Hence $\operatorname{deg}\left(f^{i_{k}}: \mathcal{K} \rightarrow \mathcal{K}_{i_{k}}\right)$ is at most 64 , so that $i_{k} \leq 6 p, k=1, \ldots, m$.

On the other hand, $\mathcal{K}_{i_{k}}$ is contained in a puzzle piece in $R$ which is mapped under $f^{i_{k+1}-i_{k}}$ onto $Y^{0}$ with degree at most $2^{m \mathbf{n}}$. It follows by the Telescope Lemma 2.2 that there is a puzzle piece $\Upsilon_{k} \subset E^{\chi-1}$ which is mapped under $f^{i_{k}}$ onto $Y^{0}$ with degree at most $2^{\bar{\chi}+k m n} \leq$ $2^{\bar{\chi}+m^{2} \mathbf{n}} \equiv D$.

We would like to apply the Covering Lemma to the corresponding map

$$
\mathbf{f}^{i_{k}}:\left(\mathbf{\Upsilon}_{k}, \mathcal{K}\right) \rightarrow\left(\mathbf{Y}^{0}, \mathcal{K}_{i_{k}}\right)
$$

of degree at most $D$. To this end we need collars around $\mathcal{K}_{i_{k}}$. Let $\Omega$ be the critical pullback of $E^{\chi}$ under $f^{6 p}$. Then we let $\Lambda_{k}^{\prime}=f^{i_{k}}(\Omega)$. Since the moments $i_{k}$ are pairwise non-congruent $\bmod p$ and $i_{k} \leq 6 p$, the puzzle pieces $\Lambda_{k}^{\prime}$ are contained in different domains of the orbit $f^{t}\left(E^{\chi}\right), t=0,1 \ldots, p-1$. Hence they are pairwise disjoint. Moreover, by Lemma 3.5,

$$
\bmod \left(\boldsymbol{\Lambda}_{k}^{\prime}, \mathcal{K}_{i_{k}}\right) \geq \bmod (\boldsymbol{\Omega}, \mathcal{K})=\frac{1}{4} \bmod \left(\mathbf{E}^{\chi-1}, \mathcal{K}\right) \geq \frac{1}{4} \bmod \left(\boldsymbol{\Upsilon}_{k}, \mathcal{K}\right) .
$$

This provides us with the desired Collar Assumption. By the Covering Lemma,

$$
\bmod \left(\mathbf{Y}^{0}, \mathcal{K}_{i_{k}}\right) \leq C 2^{14} \bmod \left(\mathbf{\Upsilon}_{k}, \mathcal{K}\right) \leq C 2^{14} \bmod \left(\mathbf{E}^{\chi-1}, \mathcal{K}\right)
$$

The last two estimates show that the Quasi-Additivity Law is applicable to the family of islands $\mathcal{K}_{i_{k}}$ in $\mathbf{Y}^{0}$ (with $\eta^{-1}=C 2^{16}$ ):

$$
\bmod \left(Y^{0}, R\right) \leq \frac{C^{3} 2^{30} \bmod \left(\mathbf{E}^{\chi-1}, \mathcal{K}\right)}{m} \leq \rho \bmod \left(\mathbf{E}^{\chi-1}, \mathcal{K}\right),
$$

provided $\bmod \left(\mathbf{E}^{\chi-1}, \mathcal{K}\right)<\varepsilon(D)=\varepsilon(\mathbf{n} ; \bar{\chi}, \rho)$ and we are done.

\subsection{Many consecutive returns to $L$}

Here the set-up is the same as in the previous section, but we will assume that there is a gap in returns of the little Julia sets to $R$ :

Lemma 5.5. - Let $\rho, \bar{\chi}, m$, and $p$ be as in Lemma 5.4. Assume there is $k \leq m$ such that

$$
i_{k+1}-i_{k}>m \mathbf{q n} \text {. }
$$

If $\chi \leq \bar{\chi}$ while $p \geq \underline{p}$, then

$$
\bmod \left(Z^{0}, L\right) \leq \rho \bmod \left(\mathbf{E}^{\chi-1}, \mathcal{K}\right)
$$

provided $\bmod \left(\mathbf{E}^{\chi-1}, \mathcal{K}\right)<\varepsilon(\mathbf{n} ; \mathbf{q}, \bar{\chi}, \rho)$. 
Proof. - Under our assumption (5.7) the Julia set returns frequently to $L$ :

$$
\mathcal{K}_{i_{k}+j \mathbf{q n}} \subset L, \quad j=1, \ldots, m .
$$

Let $P_{j} \ni f^{i_{k}} z$ be the pullback of $Z^{0}$ under $f^{j \mathbf{q n}}$. By Lemma 2.3 and the Telescope Lemma, $P_{j} \subset Y^{0}$.

Let $\Upsilon_{j}$ be the further pullback of $P_{j}$ under $f^{i_{k}}$, and let

$$
\Psi_{j}=f^{i_{k}+j \mathbf{q n}}: \Upsilon_{j} \rightarrow Z^{0} .
$$

Then $\Upsilon_{j} \subset E^{\chi-1}$ and $\operatorname{deg} \Psi_{j} \leq 2^{\bar{\chi}+\underline{p}}$.

The rest of the argument is the same as for Lemma 5.4: the Covering Lemma implies that for $j=1, \ldots, m$,

$$
\bmod \left(\mathbf{Z}_{0}, \mathcal{K}_{i_{k}+j \mathbf{q n}}\right) \leq C 2^{14} \bmod \left(\mathbf{\Upsilon}_{k}, \mathcal{K}\right) \leq C 2^{14} \bmod \left(\mathbf{E}^{\chi-1}, \mathcal{K}\right)
$$

and by the Quasi-Additivity Law,

$$
\bmod \left(Z^{0}, L\right) \leq \frac{C^{3} 2^{30} \bmod \left(\mathbf{E}^{\chi-1}, \mathcal{K}\right)}{m} \leq \rho \bmod \left(\mathbf{E}^{\chi-1}, \mathcal{K}\right)
$$

Note that by symmetry, $\bmod \left(Z^{0}, L\right)=\bmod \left(Y^{0}, R\right)$. Putting together Lemmas 5.3, 5.4 and 5.5, we obtain:

COROllary 5.6. - For any parameters $\mathbf{q}, \mathbf{n}$ of a decoration and any $\rho>0$, there exist $\underline{p} \in \mathbb{N}$ and $\varepsilon>0$ such that

$$
\bmod \left(Y^{0}, R\right) \leq \rho \bmod \left(\mathbf{E}^{\chi-1}, \mathcal{K}\right)
$$

provided $p \geq \underline{p}$ and $\bmod \left(\mathbf{E}^{\chi-1}, \mathcal{K}\right)<\varepsilon$.

\subsection{Comparison of $\bmod \left(Y^{0}, R\right)$ with $\mathbf{d}_{Y^{1}}\left(\alpha, \alpha^{\prime}\right)$}

Let

$$
\mu:=\min (\bmod (U, K), 1 / 2)
$$

\section{LEMMA 5.7}

$$
\mathbf{d}_{Y^{1}}\left(\alpha, \alpha^{\prime}\right) \leq \bmod \left(Y^{0}, R\right) \ominus \frac{1}{4} \mu .
$$

Proof. - Let $\tilde{\mathbf{Y}}^{0}=\mathbf{Y}^{0}(1)$. The boundary of $\tilde{\mathbf{Y}}^{0}$ consists of two parts (see the end of $\S 3.2$ ): the $J$-boundary $\partial_{J} \tilde{\mathbf{Y}}^{0}=\xi \cup \eta$ attached to $\alpha$ and the outer $\operatorname{arc} \lambda=\partial_{O} \tilde{\mathbf{Y}}^{0}$ that covers the ideal boundary of $\mathbf{U}$. Let $\mathcal{G}^{h}$ stand for the family of curves in the annulus $\tilde{\mathbf{Y}}^{0} \backslash K_{R}$ connecting $K_{R}$ to the $J$-boundary, while $\mathcal{G}^{v}$ stands for the family of curves in the same annulus connecting $K_{R}$ to $\lambda$. By the Parallel Law,

$$
\mathcal{L}\left(\mathcal{G}^{h}\right) \leq \bmod \left(\tilde{Y}^{0}, R\right) \ominus \mathcal{L}\left(\mathcal{G}^{v}\right) .
$$

Let $\Pi$ stand for the rectangle uniformizing $\tilde{\mathbf{Y}}^{0} \backslash K_{Y^{0}}$ whose horizontal sides correspond to $K_{Y^{0}}$ and $\lambda$, and vertical sides correspond to $\xi$ and $\eta$. We let $\omega$ be the horizontal side of $\Pi$ corresponding to $K_{Y^{0}}$. Since any curve of the family $\mathcal{G}^{v}$ overflows some curve connecting $K_{Y^{0}}$ to $\lambda$ in $\tilde{\mathbf{Y}}^{0} \backslash K_{Y^{0}}$ (and thus representing a vertical curve in $\Pi$ ), we have:

$$
\mathcal{L}\left(\mathcal{G}^{v}\right) \geq \bmod \Pi \text {. }
$$

$4^{\text {e }}$ SÉRIE - TOME $41-2008-\mathrm{N}^{\mathrm{o}} 1$ 
But by definition of the pseudo-puzzle, the domain $\tilde{\mathbf{Y}}^{0} \backslash K_{Y^{0}}$ covers the annulus $\mathbf{U} \backslash K$ extending to an embedding on $K_{Y^{0}}$. Let us uniformize $\mathbf{U} \backslash K$ by a round annulus $\mathbb{A}$. It follows that the rectangle $\Pi$ covers $\mathbb{A}$ in such a way that $\omega \subset \partial \Pi$ is embedded into $\partial \mathbb{A}$. By Lemma 6.6 from the Appendix,

$$
\bmod \Pi \geq \frac{\mu}{4}
$$

Putting the above three estimates together, we obtain:

$$
\mathcal{L}\left(\mathcal{G}^{h}\right) \leq \bmod \left(\tilde{Y}^{0}, R\right) \ominus \frac{\mu}{4} .
$$

On the other hand, let us consider the family $\mathcal{H}$ of horizontal curves in the puzzle piece $\mathbf{Y}^{1}$ connecting $\partial_{\alpha} \mathbf{Y}^{1}$ to $\partial_{\alpha^{\prime}} \mathbf{Y}^{1}$. Let $\phi: \mathbf{Y}^{1} \rightarrow \tilde{\mathbf{Y}}^{0}$ be the natural immersion. Under $\phi$, the boundary $\partial_{\alpha} \mathbf{Y}^{1}$ is mapped homeomorphically onto $\partial_{\alpha} \tilde{\mathbf{Y}}^{0}$. It follows that any curve $\gamma$ of $\mathcal{G}^{h}$ contains an arc that can be lifted by $\phi$ to some curve of $\mathcal{H}$. Indeed, orient $\gamma$ so that it begins on $\partial_{\alpha} \tilde{\mathbf{Y}}^{0}$. Then a maximal lift of $\gamma$ that begins on $\partial_{\alpha} \mathbf{Y}^{1}$ must end on $\partial_{\alpha^{\prime}} \mathbf{Y}^{1}$.

By Corollary 6.2, $\mathcal{L}(\mathcal{H}) \leq \mathcal{L}\left(\mathcal{G}^{h}\right)$. Together with (5.8), this yields the desired inequality.

\subsection{Skipping over}

In this section we will show that not many curves can skip some piece of the Julia set.

Let $Y \in \mathcal{Y}^{m}(l)$ be a geometric puzzle piece of bidepth $(m, l)$, and let $A$ be a component of $Y \backslash K_{Y}$. Let $\partial_{J} \hat{A}=\partial_{J} \mathbf{Y} \cap \hat{A}$. Recall that it consists of two components. Let $\mathcal{C}_{A}$ stand for the family of curves in $\hat{A}$ connecting different components of $\partial_{J} \hat{A}$, and let

$$
\mathbf{d}_{A}=\mathcal{L}\left(\mathcal{C}_{A}\right) \text {. }
$$

Let $\frac{1}{2 \pi} \log r=2^{-(m+\mathbf{q})} \mu$. Then the annulus $\mathbf{U}^{m+\mathbf{q}} \backslash K$ can be uniformized by the round annulus $\mathbb{A}(1, r)$, and under this uniformization, the set $K_{Y}$ gets represented on the unit circle $\mathbb{T}$ as the union of arcs $\omega_{i}$ of length

$$
\left|\omega_{i}\right| \geq 2 \pi \cdot 2^{-(\mathbf{q}+m)} .
$$

Indeed, the covering map $\mathbf{f}^{m+\mathbf{q}}: \mathbf{U}^{m+\mathbf{q}} \backslash K \rightarrow \mathbf{U} \backslash K$ is turned into $z \mapsto z^{2^{m+\mathbf{q}}}$ under the above uniformization of $\mathbf{U}^{m+\mathbf{q}} \backslash K$ and the uniformization of $\mathbf{U} \backslash K$ by $\mathbb{A}\left(1, e^{2 \pi \mu}\right)$ (appropriately normalized). Since under this map, every arc $\omega_{i}$ covers the whole circle, the length of $\omega_{i}$ is at least $2 \pi$ times its combinatorial length (2.2).

Lemma 5.8. - Let $Y \in \mathcal{Y}^{m}(m+\mathbf{q})$ be a geometric puzzle piece of bidepth $(m, m+\mathbf{q})$, and let $A$ be a component of $Y \backslash K_{Y}$. Then

$$
\mathbf{d}_{A} \geq \frac{1}{\mu} .
$$

Proof. - We can uniformize $\hat{A}$ by the horizontal strip $\Pi=\Pi\left(2^{-(m+\mathbf{q})} \mu\right)$ in such a way that the upper boundary of $\Pi$ covers the $O$-boundary of $\mathbf{U}^{m+\mathbf{q}}$, and the group of deck transformations is generated by the translation $z \mapsto z+1$. By (5.9), the Julia set $K_{Y} \subset \partial A$ is represented as an interval $I$ on $\mathbb{R}$ of length at least $2^{-(\mathbf{q}+m)}$.

Let us view $\Pi$ as a quadrilateral with horizontal sides $I$ and the top of $\Pi$. Then

$$
\mathcal{L}\left(\mathcal{C}_{A}\right)=\frac{1}{\bmod \Pi} \geq \frac{1}{\mu},
$$


where the last estimate comes from the simple right-hand side estimate of Lemma 6.5, and we are done.

Lemma 5.9. - Let $Y \in \mathcal{Y}^{m}(0)$ be a geometric puzzle piece of bidepth $(m, 0)$, and let $A$ be a connected component of $Y \backslash K_{Y}$. Then

$$
\mathbf{d}_{A} \geq \frac{\mu}{2^{m+\mathbf{q}+2}} .
$$

Proof. - Let $A(m+\mathbf{q})$ be the component of $Y(m+\mathbf{q}) \backslash K_{Y}$ contained in $A$, and let $\phi: \hat{A}(m+\mathbf{q}) \rightarrow \hat{A}$ be the natural immersion. It extends to the identity on $K_{Y} \cap \partial \hat{A}(m+\mathbf{q})$.

Let us realize $\hat{A}$ as the strip $\Pi(\mu)$ that covers $\mathbf{U} \backslash K$, with the group of deck transformations generated by $z \mapsto z+1$. Let us consider the interval $I \subset \mathbb{R}$ representing $K_{Y} \cap \partial A$, and let $J$ be the left-adjacent interval of length 1 .

Let us orient the curves $\gamma \in \mathcal{C}_{A}$ so that they begin on the left-hand side of $I$. Then any curve $\gamma \in \mathcal{C}_{A}$ contains the maximal initial arc $\gamma^{\prime}$ that can be lifted by $\phi$ to a curve $\gamma^{*}$ in $\hat{A}(m+\mathbf{q})$. Accordingly, we can split the family of curves $\gamma \in \mathcal{C}_{A}$ into three subfamilies:

- $\mathcal{H}_{1}$ consists of the curves $\gamma$ such that $\gamma^{\prime}=\gamma$; then $\gamma^{*} \in \mathcal{C}_{A(m+\mathbf{q})}$;

- $\mathcal{H}_{2}$ consist of the curves that begin in $J$ and whose lift $\gamma^{*}$ terminates on the $O$-boundary of $\hat{A}(m+\mathbf{q})$;

- $\mathcal{H}_{3}$ consists of the curves that begin on the left-hand side of $J$.

Let us estimate the extremal length of each of these families.

Since $\mathcal{H}_{1}=\phi\left(\mathcal{C}_{A(m+\mathbf{q})}\right)$,

$$
\mathcal{L}\left(\mathcal{H}_{1}\right) \geq \mathcal{L}\left(\mathcal{C}_{A(m+\mathbf{q})}\right) \geq \frac{1}{\mu}
$$

where the first estimate follows from Lemma 6.1, and the second follows from Lemma 5.8.

Let $\mathcal{T}$ be the family of curves in $\hat{A}(m+\mathbf{q})$ that begin on $J$ and end on the $O$-boundary of $\hat{A}(m+\mathbf{q})$. By Corollary 6.2 and Lemma 6.5,

$$
\mathcal{L}\left(\mathcal{H}_{2}\right) \geq \mathcal{L}(\mathcal{T}) \geq \frac{\mu}{2^{m+\mathbf{q}+1}}
$$

To estimate the extremal length of $\mathcal{H}_{3}$, endow the rectangle $Q=J \times[0, \mu] \subset \Pi(\mu)$ with the Euclidean metric $\lambda$. Since any curve $\gamma \in \mathcal{H}_{3}$ horizontally overflows $Q$, it has $\lambda$-length at least 1 . Hence

$$
\mathcal{L}\left(\mathcal{H}_{3}\right) \geq \frac{1}{\operatorname{area} Q}=\frac{1}{\mu}
$$

Incorporating the last three estimates into the Parallel Law, we obtain the desired:

$$
\mathcal{L}\left(\mathcal{C}_{Y}^{\varepsilon}\right) \geq \frac{1}{\mu+2^{m+\mathbf{q}+1} \mu^{-1}+\mu} \geq \frac{\mu}{2^{m+\mathbf{q}+2}} .
$$

Let us consider two vertices, $v$ and $w$, of a geometric puzzle piece $Y$. Let $Z \subset Y$ be a puzzle piece of depth $m$ that separates $v$ from $w$. We say that a multicurve in $\mathbf{Y}$ connecting $\partial^{v} \mathbf{Y}$ to $\partial^{w} \mathbf{Y}$ skips over $K_{Z}$ if one of its components does not cross $K_{Z}$.

$4^{\mathrm{e}}$ SÉRIE - TOME $41-2008-\mathrm{N}^{\mathrm{o}} 1$ 
COROLlaRY 5.10. - Under the above circumstances, let $\mathcal{T}$ be the family of multicurves in $\mathbf{Y}$ connecting $\partial^{v} \mathbf{Y}$ to $\partial^{w} \mathbf{Y}$ that skip over $K_{Z}$. Then

$$
\mathcal{L}(\mathcal{T}) \geq \frac{\mu}{2^{2 m+\mathbf{q}+2}}
$$

Proof. - The piece $Z$ has at most $2^{m}$ components $A_{i}$ of $Z \backslash K_{Z}$. If a multicurve $\gamma$ in $\mathbf{Y}$ skips over $K_{Z}$ then it contains an arc $\gamma^{\prime}$ that lifts to a curve $\gamma^{*}$ in some family $\mathcal{G}_{A_{j}}$. Let $\mathcal{C}_{j}$ be the corresponding subfamily of $\mathcal{T}$. By Lemma 5.9 (together with Corollary 6.2),

$$
\mathcal{L}\left(\mathcal{C}_{j}\right) \geq \mathbf{d}_{A_{j}} \geq \frac{\mu}{2^{m+\mathbf{q}+2}} .
$$

The Parallel Law concludes the proof.

Let us now consider the puzzle piece $P=Y^{(\mathbf{n}-1) \mathbf{q}+1}$, together with the corresponding pseudo-piece $\mathbf{P}$, and the family of puzzle pieces $Q^{v} \subset P$ from Lemma 2.1. Recall that $T^{v w}=$ $K_{P} \backslash\left(Q^{v} \cup Q^{w}\right)$. Given two vertices $v$ and $w$ of $P$, let $\hat{\mathcal{G}}_{P}^{v w}$ stand for the family of multicurves in $\mathbf{P}$ connecting $\partial_{v} \mathbf{P}$ to $\partial_{w} \mathbf{P}$ that do not skip over $T_{v w}$. By Corollary 5.10,

$$
\mathcal{L}\left(\mathcal{G} \backslash \hat{\mathcal{G}}_{P}^{v w}\right) \geq C^{-1} \mu
$$

where, here and below, $C$ stands for a constant that depends only on $\mathbf{q}$ and $\mathbf{n}$.

\subsection{Separation between $L$ and $R$}

In this section we will show that the modulus $\mathbf{d}_{Y^{1}}\left(\alpha, \alpha^{\prime}\right)$ that measures the extremal distance between $L$ and $R$ is comparable with $\mu$.

Let $Y$ be a geometric puzzle piece. For two vertices $v$ and $w$ of $Y$, we let

$$
\mathbf{W}_{Y}(v, w)=\mathcal{W}\left(\mathcal{G}_{Y}^{v w}\right) .
$$

We define the pseudo-conductance of $Y$ as

$$
\mathbf{W}_{Y}=\sup _{v, w} \mathbf{W}_{Y}(v, w),
$$

where the supremum is taken over all pairs of the vertices of $Y$.

Lemma 5.11. - For the puzzle piece $P=Y^{(\mathbf{n}-1) \mathbf{q}+1}$ we have:

$$
W_{P} \leq \frac{C}{\mu} .
$$

Proof. - Along with the above conductance of $P$, let us consider

$$
\hat{\mathbf{W}}_{P}(v, w)=\mathcal{W}\left(\hat{\mathcal{G}}_{P}^{v w}\right) ; \quad \hat{\mathbf{W}}_{P}=\sup _{v, w} \hat{\mathbf{W}}_{P}(v, w)
$$

By (5.10),

$$
\mathbf{W}_{P} \leq \hat{\mathbf{W}}_{P}+\frac{C}{\mu} .
$$

Take a pair of vertices, $v$ and $w$. Let $Q^{v} \cap T^{v w}=\left\{v^{\prime}\right\}$ and $Q^{w} \cap T^{v w}=\left\{w^{\prime}\right\}$. Recall that depth of the puzzle pieces $Q^{v}$ and $Q^{w}$ is equal to $r=(2 \mathbf{n}-1) \mathbf{q}+1$, and so depends only on $\mathbf{q}$ and $\mathbf{n}$. Let $\mathcal{E}^{r}$ be the lift of the equipotential of level $r$ to $\mathbf{P}$.

For any horizontal multicurve $\gamma \in \hat{\mathcal{G}}_{P}^{v w}$, one of the following two possibilities can occur: 
1. $\gamma$ crosses the equipotential $\mathcal{E}^{r}$, and hence it contains an $\operatorname{arc} \gamma^{\prime}$ connecting $\mathcal{E}^{r}$ to $T^{v w}$; By Lemma 6.6 and the Parallel Law, the width of this family of curves is bounded by $2^{r+\mathbf{n}} / \mu$ (here $2^{\mathbf{n}-1}$ is a bound on the number of connected components of $P \backslash K_{P}$ );

2. $\gamma$ contains two disjoint multicurves, $\delta^{v}$ and $\delta^{w}$, that do not cross $\mathcal{E}^{r}$ and such that $\delta^{v}$ connects $\partial^{v} \mathbf{P}$ to $T^{v w}$, while $\delta^{w}$ connects $T^{v w}$ to $\partial^{w} \mathbf{P}$. Then $\delta^{v}$ contains a multicurve that can be lifted to a horizontal multicurve in $\mathbf{Q}^{v}$ connecting $\partial^{v} \mathbf{Q}^{v}$ to $\partial^{v^{\prime}} \mathbf{Q}^{v}$, and similarly for $\delta^{w}$.

By the Series and Parallel Laws,

$$
\hat{\mathbf{W}}_{P}(v, w) \leq \mathbf{W}_{Q^{v}}\left(v, v^{\prime}\right) \oplus \mathbf{W}_{Q^{w}}\left(w, w^{\prime}\right)+\frac{2^{r+\mathbf{n}}}{\mu} \leq \mathbf{W}_{Q^{v}} \oplus \mathbf{W}_{Q^{w}}+\frac{2^{r+\mathbf{n}}}{\mu} .
$$

But $\mathbf{W}\left(Q^{v}\right)=\mathbf{W}\left(Q^{w}\right)=\mathbf{W}(P)$ since $Q^{v}$ and $Q^{w}$ are univalent pullbacks of $P$. Hence

$$
\mathbf{W}_{Q^{v}} \oplus \mathbf{W}_{Q^{w}} \leq \frac{1}{2} \mathbf{W}_{P} .
$$

Putting the last two estimates together and taking the supremum over all pairs of vertices $(v, w)$ of $P$, we conclude that

$$
\hat{\mathbf{W}}_{P} \leq \frac{1}{2} \mathbf{W}_{P}+\frac{2^{r+\mathbf{n}}}{\mu}
$$

Together with (5.11) it yields:

$$
\mathbf{W}_{P} \leq \frac{1}{2} \mathbf{W}_{P}+\frac{C}{\mu}
$$

and the conclusion follows.

Proposition 5.12. - We have: $\mathbf{d}_{Y^{1}}\left(\alpha, \alpha^{\prime}\right) \geq C^{-1} \mu$.

Proof. - Since the map $f^{(\mathbf{n}-1) \mathbf{q}}: \mathbf{P} \rightarrow \mathbf{Y}^{1}$ is a branched covering that maps $\partial_{0} \mathbf{P}$ to $\partial_{0} \mathbf{Y}^{1}$, any curve $\gamma \in \mathcal{G}_{Y^{1}}^{\alpha \alpha^{\prime}}$ can be lifted to a curve $\gamma^{*} \in \cup \mathcal{G}_{P}^{v w}$, where the union is taken over all pairs of vertices of $P$. Hence

$$
\mathcal{L}\left(\mathcal{G}_{Y^{1}}^{\alpha \alpha^{\prime}}\right) \geq \bigoplus_{v, w} \mathcal{L}\left(\mathcal{G}_{P}^{v w}\right) \geq \frac{1}{N \mathbf{W}_{P}}
$$

where $N$ is the number of pairs $(v, w)$. The conclusion follows.

Lemma 5.7 and Proposition 5.12 imply:

Corollary 5.13. - We have: $\bmod \left(Y^{0}, R\right) \geq C^{-1} \mu$.

Corollary 5.14. - Let $f:(\mathbf{U}, K) \rightarrow(\mathbf{U}, K)$ be a renormalizable $\psi$-quadratic-like map with decoration parameters $(\mathbf{q}, \mathbf{n})$, and let $f^{\prime}=f^{p}:\left(\mathbf{U}^{\prime}, K^{\prime}\right) \rightarrow\left(\mathbf{U}^{\prime}, K^{\prime}\right)$ be its first renormalization. Then

$$
\min \{\bmod (\mathbf{U}, K), 1 / 2\} \leq C \bmod \left(\mathbf{U}^{\prime}, K^{\prime}\right)
$$

where $C=C(\mathbf{q}, \mathbf{n})$.

Proof. - This follows from Lemma 5.3 and Corollary 5.13 by noticing that $\left(\mathbf{E}^{\chi-1}, \mathcal{K}\right)=$ $\left(\mathbf{U}^{\prime}, K^{\prime}\right)$.

$4^{\text {e }}$ SÉRIE - TOME $41-2008-\mathrm{N}^{\mathrm{o}} 1$ 


\subsection{Conclusion}

Everything is now prepared for the main results. Corollary 5.6 and Corollary 5.13 imply:

THEOREM 5.15 (Improving of the moduli: bounded decoration parameters)

For any parameters $\overline{\mathbf{q}}, \overline{\mathbf{n}}$ and any $\rho>0$, there exist $p \in \mathbb{N}$ and $\varepsilon>0$ with the following property. Let $f:(\mathbf{U}, K) \rightarrow(\mathbf{U}, K)$ be a renormalizable $\bar{\psi}$-quadratic-like map with decoration parameters $(\mathbf{q}, \mathbf{n}) \leq(\overline{\mathbf{q}}, \overline{\mathbf{n}})$, and let $f^{\prime}=f^{p}:\left(\mathbf{U}^{\prime}, K^{\prime}\right) \rightarrow\left(\mathbf{U}^{\prime}, K^{\prime}\right)$ be its first renormalization. Then

$$
\left\{p \geq \underline{p} \text { and } \bmod \left(\mathbf{U}^{\prime}, K^{\prime}\right)<\varepsilon\right\} \Rightarrow \bmod (\mathbf{U}, K)<\rho \bmod \left(\mathbf{U}^{\prime}, K^{\prime}\right) .
$$

REMARK 5.1. - The logic of this theorem can be adjusted so that it would sound more like an "improvement in the future" rather than "worsening in the past":

For any parameters $\overline{\mathbf{q}}, \overline{\mathbf{n}}$ of a Misuirewicz limb, there exist $\underline{p} \in \mathbb{N}$ and $\varepsilon>0$ such that

$$
\bmod \left(\mathbf{U}^{\prime}, K^{\prime}\right) \geq 2 \bmod (\mathbf{U}, K)
$$

provided $p \geq \underline{p}$ and $\bmod (\mathbf{U}, K)<\varepsilon / 2$.

Theorem 5.15, together with Lemma 3.1, implies Theorem 1.2 from the Introduction.

To derive the Main Theorem, we will combine Theorem 5.15 with the following result (Theorem 9.1 from [6]):

THEOREM 5.16 (Improving of the moduli: bounded period). - For any $\rho \in(0,1)$, there exists $\underline{p}=\underline{p}(\rho)$ such that for any $\bar{p} \geq \underline{p}$, there exists $\varepsilon=\varepsilon(\bar{p})>0$ with the following property. Let $f:(\mathbf{U}, K) \rightarrow(\mathbf{U}, K)$ be a primitively renormalizable $\psi$-quadratic-like map, and let $f^{\prime}=$ $f^{p}=\left(\mathbf{U}^{\prime}, K^{\prime}\right) \rightarrow\left(\mathbf{U}^{\prime}, K^{\prime}\right)$ be the corresponding renormalization. Then

$$
\left\{\underline{p} \leq p \leq \bar{p} \text { and } \bmod \left(\mathbf{U}^{\prime} \backslash K^{\prime}\right)<\varepsilon\right\} \Rightarrow \bmod (\mathbf{U} \backslash K)<\rho \bmod \left(\mathbf{U}^{\prime} \backslash K^{\prime}\right) .
$$

REMARK 5.2. - Unlike Theorem 5.15, in Theorem 5.16 the map $f^{\prime}$ is not necessarily the first renormalization of $f$. On the other hand, in Theorem 5.16, the scale $\varepsilon$ depends on the upper bound $\bar{p}$, while in Theorem 5.15 it does not.

We say that an infinitely renormalizable $\psi$-ql map $f$ belongs to the decoration class $(\overline{\mathbf{q}}, \overline{\mathbf{n}})$ if the decoration parameters $\left(\overline{\mathbf{q}}_{n}, \overline{\mathbf{n}}_{n}\right)$ of the renormalizations $R^{n} f$ are all bounded by $(\overline{\mathbf{q}}, \overline{\mathbf{n}})$.

Let us now put the above two theorems together:

Corollary 5.17. - For any $(\overline{\mathbf{q}}, \overline{\mathbf{n}})$, there exist an $\varepsilon>0$ and $l \in \mathbb{N}$ with the following property. For any infinitely renormalizable $\psi$-ql map $f$ of decoration class $(\overline{\mathbf{q}}, \overline{\mathbf{n}})$ with renormalizations $R^{n} f:\left(\mathbf{U}_{n}, K_{n}\right) \rightarrow\left(\mathbf{U}_{n}, K_{n}\right)$, if $\bmod \left(\mathbf{U}_{n} \backslash K_{n}\right)<\varepsilon, n \geq l$, then $\bmod \left(\mathbf{U}_{n-l} \backslash K_{n-l}\right)<$ $\bmod \left(\mathbf{U}_{n} \backslash K_{n}\right) / 2$. 
Proof. - Given an infinitely renormalizable $\psi$-ql map $f$ with renormalizations $R^{n} f$ : $\left(\mathbf{U}_{n}, K_{n}\right) \rightarrow\left(\mathbf{U}_{n} \backslash K_{n}\right)$, we let $\mu_{n}(f)=\bmod \left(\mathbf{U}_{n}, K_{n}\right)$. Arguing by contradiction, we find a sequence $f_{i}$ of infinitely renormalizable $\psi$-ql maps of decoration class $(\overline{\mathbf{q}}, \overline{\mathbf{n}})$ and sequences $\varepsilon_{i} \rightarrow 0$ and $n(i) \rightarrow \infty$ such that:

P1: $\mu_{n(i)}\left(f_{i}\right)<\varepsilon_{i}$;

P2: $\mu_{n(i)}\left(f_{i}\right)<2 \mu_{k}\left(f_{i}\right), k=0,1, \ldots, n(i)-1$.

Let $R^{n} f_{i}$ be the renormalization of $R^{n-1} f_{i}$ with period $p_{n}\left(f_{i}\right)$. Applying then the diagonal process, we can also assume the following property:

P3: $p_{n(i)-s}\left(f_{i}\right) \rightarrow \pi_{s} \in \mathbb{N} \cup\{\infty\}$ for $s=0,1, \ldots$

We let $\bar{s} \in \mathbb{Z}_{\geq 0} \cup\{\infty\}$ be the first moment for which $\pi_{s}=\infty$ (with understanding that $\bar{s}=\infty$ if such a moment does not exist).

Let us consider two cases:

Case 1: $\bar{s}<\infty$. Applying consecutively Corollary 5.14, we conclude that for sufficiently big $i$,

$$
\mu_{n(i)-s}\left(f_{i}\right) \leq C^{s} \mu_{n(i)}\left(f_{i}\right), \quad s \leq \bar{s} .
$$

Let $\rho \in\left(0,1 / 2 C^{\bar{s}}\right)$. By Theorem 5.15, for all sufficiently big $i$,

$$
\mu_{n(i)-\bar{s}-1}\left(f_{i}\right) \leq \rho \mu_{n(i)-\bar{s}}\left(f_{i}\right) .
$$

Putting the last two estimates together, we conclude that for all sufficiently big $i$,

$$
\mu_{n(i)-\bar{s}-1}\left(f_{i}\right)<\frac{1}{2} \mu_{n(i)}\left(f_{i}\right)
$$

contradicting assumption (P2).

Case 2: $\bar{s}=\infty$. Take an $s$ such that

$$
\bar{p} \equiv \pi_{0} \pi_{1} \ldots \pi_{s}>\underline{p},
$$

where $\underline{p}=\underline{p}(1 / 2)$ comes from Theorem 5.16. By this theorem, for sufficiently big $i$,

$$
\mu_{n(i)-s-1}(i)<\frac{1}{2} \mu_{n(i)}(i),
$$

contradicting again assumption (P2).

We are ready to prove the Main Theorem, in an important refined version. We say that a family $\mathcal{M}$ of little Mandelbrot copies (and the corresponding renormalization combinatorics) has beau ${ }^{(6)}$ a priori bounds if there exist an $\varepsilon=\varepsilon(\mathcal{M})>0$ and a function $N: \mathbb{R}_{+} \rightarrow \mathbb{N}$ with the following property. Let $f: U \rightarrow V$ be a quadratic-like map with $\bmod (V \backslash U) \geq$ $\delta>0$ that is at least $N=N(\delta)$ times renormalizable. Then for any $n \geq N$, the $n$-fold renormalization of $f$ can be represented by a quadratic-like map $R^{n} f: U_{n} \rightarrow V_{n}$ with $\bmod \left(V_{n} \backslash U_{n}\right) \geq \varepsilon$.

Beau Bounds (Refined Main Theorem). - For any parameters $(\overline{\mathbf{q}}, \overline{\mathbf{n}})$, the family of renormalization combinatorics of decoration class $(\overline{\mathbf{q}}, \overline{\mathbf{n}})$ has beau a priori bounds.

(6) According to Dennis Sullivan, "beau" stands for "bounded and eventually universal".

$4^{\mathrm{e}}$ SÉRIE - TOME $41-2008-\mathrm{N}^{\mathrm{o}} 1$ 
Proof. - Let $\varepsilon>0$ and $l$ come from Corollary 5.17, and $C>0$ come from Corollary 5.14. We will use notation $\mu_{n}(f)$ from the proof of Corollary 5.17. Assume that for some $\delta>0$, there is a sequence of $\psi$-ql maps $f_{i}$ in question with $\mu_{0}\left(f_{i}\right) \geq \delta$, while $\mu_{n(i)}\left(f_{i}\right)<\varepsilon$, where $n(i) \rightarrow \infty$. Let $n(i)=k_{i} l+r_{i}$ where $0 \leq r_{i}<l$. Then by Corollaries 5.17 and 5.14,

$$
\mu_{0}\left(f_{i}\right) \leq C^{r_{i}} \varepsilon / 2^{k_{i}} \rightarrow 0 \quad \text { as } i \rightarrow \infty \text {. }
$$

This contradiction proves the beau bounds for the moduli $\mu_{n}(f)$ of $\psi$-ql maps. The beau bounds for ordinary quadratic-like maps follow by Lemma 3.1.

\section{Appendix: Extremal length and width}

Given a family of curves $\mathcal{G}$ on a Riemann surface $S$ and a conformal metric $\mu$ on $S$, we let $\mu(\gamma)$ be the $\mu$-length of a curve $\gamma \in \Gamma, \mu(\Gamma)$ be the infimum of these lengths, area ${ }_{\mu}$ be the corresponding measure, and $\mathcal{L}(\mathcal{G})$ and $\mathcal{W}(\mathcal{G})=\mathcal{L}(\mathcal{G})^{-1}$ be respectively the extremal length and width of $\mathcal{G}$ : see [1] or the Appendices [8,6] for the precise definitions. The most basic properties of these conformal invariants, the Parallel and Series Laws can also be found in these sources.

\subsection{Transformation rules}

Both extremal length and extremal width are conformal invariants. More generally, we have:

Lemma 6.1. - Let $f: U \rightarrow V$ be a holomorphic map between two Riemann surfaces, and let $\mathcal{G}$ be a family of curves on $U$. Then

$$
\mathcal{L}(f(\mathcal{G})) \geq \mathcal{L}(\mathcal{G})
$$

See Lemma 4.1 of [8] for a proof.

COROLlary 6.2. - Under the circumstances of the previous lemma, let $\mathcal{H}$ be a family of curves in $V$ satisfying the following lifting property: any curve $\gamma \in \mathcal{H}$ contains an arc that lifts to some curve in $\mathcal{G}$. Then $\mathcal{L}(\mathcal{H}) \geq \mathcal{L}(\mathcal{G})$.

See Corollary 10.3 of [6] for a proof.

Given a compact subset $K \subset \operatorname{int} U$, the extremal distance

$$
\mathcal{L}(U, K) \equiv \bmod (U, K)
$$

(between $\partial U$ and $K$ ) is defined as $\mathcal{L}(\mathcal{G})$, where $\mathcal{G}$ is the family of curves connecting $\partial U$ and $K$. In case when $U$ is a topological disk and $K$ is connected, we obtain the usual modulus $\bmod (U \backslash K)$ of the annulus $U \backslash K$. We let $\mathcal{W}(U, K)=\mathcal{L}^{-1}(U, K)$.

Lemma 6.3. - Let $f: U \rightarrow V$ be a branched covering between two compact Riemann surfaces with boundary. Let $A$ be a subsurface of finite type in $U, B=f(A)$, and assume that $f: A \rightarrow B$ is a branched covering of degree $d$. Then

$$
\bmod (V, B) \geq d \bmod (U, A)
$$

See Lemma 4.3 of [8] for a proof. 
Lemma 6.4. - Let $(U, A)$ and $(V, B)$ be as above, and let $f: U \backslash A \rightarrow V \backslash B$ be a branched covering of degree $N$. Then

$$
\bmod (V, B)=N \bmod (U, A)
$$

See [1] for a proof.

\subsection{Strips and quadrilaterals}

Lemma 6.5. - Let us consider a horizontal strip $\Pi(h)$ and an interval $I=(x, x+a) \subset \mathbb{R}$. We view $\Pi$ as a quadrilateral with horizontal sides $I$ and $\mathbb{R}+i h$. Then

$$
\frac{h}{2 a} \leq \bmod \Pi \leq \frac{h}{a},
$$

provided $h / a \leq 1 / 2$ or $\bmod \Pi \leq 1 / 4$ (for the left-hand side inequality).

Proof. - By definition, mod $\Pi$ is the extremal length of the family of curves connecting $I$ to $\mathbb{R}+i h$. This family contains the family $\mathcal{G}^{\prime}$ of vertical curves in the Euclidean rectangle with horizontal sides $I$ and $I+i h$. Hence $\mathcal{L}(\mathcal{G}) \leq \mathcal{L}\left(\mathcal{G}^{\prime}\right)=h / a$.

To prove the left-hand side inequality, let us consider a Euclidean rectangle $Q$ with vertices $x-h, x+a+h, x+a+h+i h, x-h+i h$ endowed with the Euclidean metric $\mu$. Any curve of $\mathcal{G}$ has $\mu$-length at least $h$. Hence

$$
\mathcal{L}(\mathcal{G}) \geq \frac{h^{2}}{\operatorname{area}_{\mu}(Q)}=\frac{t}{1+2 t}, \quad \text { where } t=h / a .
$$

We see that $\mathcal{L}(\mathcal{G}) \geq t / 2$ for $t \leq 1 / 2$, while $\mathcal{L}(\mathcal{G})>1 / 4$ otherwise. The conclusion follows.

Lemma 6.6. - Let $\Pi$ and $I$ be as in the previous lemma. Let $C=\Pi / l \mathbb{Z}$ be a cylinder covered by $\Pi$ so that $I$ is embedded into the bottom of $C$. Then

$$
\bmod \Pi \geq \frac{1}{2} \min (\bmod C, 0.5) .
$$

Proof. - Since the covering $\Pi \rightarrow C$ is an embedding on $I$, we have: $a \leq l$. Then by the previous lemma we obtain:

$$
\bmod \Pi \geq \frac{h}{2 a} \geq \frac{h}{2 l}=\frac{1}{2} \bmod C,
$$

provided $\bmod \Pi \leq 1 / 4$.

$4^{\mathrm{e}}$ SÉRIE - TOME $41-2008-\mathrm{N}^{\circ} 1$ 


\subsection{Holomorphic and embedded annuli}

Let $S$ be a hyperbolic Riemann surface with boundary with a preferred component $\sigma$ of $\partial S$. We assume that $S$ has finite topological type and is not the punctured disk. A holomorphic annulus in $\mathcal{A}$ is a holomorphic map $A: \mathbb{A}(1, r) \rightarrow S$ that extends to a homeomorphism $\phi: \mathbb{T} \rightarrow \sigma$. We let $\bmod A=\bmod \mathbb{A}(1, r)$.

The family of holomorphic annuli contains a subfamily of embedded annuli. Among embedded annuli, there is an annulus $A_{*}$ of maximal modulus, which has nice special properties. Namely, let us uniformize $A_{*}$ by a flat cylinder $C=\Pi(h) / \mathbb{Z}$. Then the quadratic differential $d z^{2}$ on $C$ is the pull-back of some quadratic differential $q$ on $S$. Moreover, the uniformization $C \rightarrow A_{*}$ extends continuously to the upper boundary $C^{+}=\mathbb{R}+i h / \mathbb{Z}$ of $C$ (minus finitely many points corresponding to the punctures of $S$ ), and induces there an equivalence relation $\tau_{k}: \alpha_{k} \rightarrow \alpha_{k}^{\prime}$, where $\left(\tau_{k}\right)$ is a finite family of isometries between pairs of disjoint arcs in $C^{+}$. The images of these arcs, $\lambda_{k}=i\left(\alpha_{k}\right)=i\left(\alpha_{k}^{\prime}\right)$, are horizontal separatricies of $q$. (It is a version of Strebel's Theorem, see e.g., [5, §11]).

Lemma 6.7. - For any holomorphic annulus $A: \mathbb{A}(1, r) \rightarrow S$, we have:

$$
\bmod A \leq 16 \bmod A_{*} .
$$

Proof. - Let us consider a family $\mathcal{G}$ of non-trivial proper curves $\gamma$ in $S$ that begin in $\sigma{ }^{(7)}$ Then any curve $\gamma \in \mathcal{G}$ contains an initial segment $\gamma^{\prime}$ that lifts to a vertical curve in $\mathbb{A}(1, r)$. By Corollary 6.2,

$$
\bmod A \leq \mathcal{L}(\mathcal{G})
$$

Let us now take any conformal metric $\mu$ on $S$, and let $l=\mu(\mathcal{G})$. For any vertical curve $\delta$ in $A_{*}$, two possibilities can occur:

1. $\delta$ ends on $\partial S$. Then $\delta \in \mathcal{G}$ and hence $\mu(\delta) \geq l$.

2. $\delta$ ends on some separatrix $i\left(\alpha_{k}\right)$. Then there is another vertical curve $\lambda$ in $A_{*}$ that ends at the same point as $\delta$. The concatenation of $\delta$ and $\lambda$ is a curve of family $\mathcal{G}$. Hence one of the curves, $\delta$ or $\lambda$, is "long", i.e., it has $\mu$-length at least $l / 2$.

It follows that at least one half of the vertical curves in $A_{*}$ are long. Let $I \subset C^{+}$be the set of endpoints of $i^{-1}$ (long curves). We can now proceed as in the classical Grötztsch estimate. By the Cauchy-Schwarz Inequality,

$$
h \operatorname{area}_{\mu}(C)=\operatorname{area}(C) \int_{C} \operatorname{area}_{\mu} d x d y \geq\left(\int_{I} d x \int_{0}^{h} \mu(x, y) d y\right)^{2} \geq\left(\frac{l}{4}\right)^{2},
$$

which implies

$$
\mathcal{L}_{\mu}(\mathcal{G})=l^{2} / \operatorname{area}_{\mu}(C) \leq 16 h=16 \bmod A_{*} .
$$

Since this is valid for any conformal metric $\mu$, we conclude that $\mathcal{L}(\mathcal{G}) \leq 16 \bmod A_{*}$. Together with (6.1), this gives us the desired estimate.

(7) "Non-trivial" means that $\gamma$ cannot be pulled to $\sigma$ through a continuous family of proper curves. 


\section{REFERENCES}

[1] L. V. Ahlfors, Conformal invariants: topics in geometric function theory, McGraw-Hill Book Co., 1973, McGraw-Hill Series in Higher Mathematics.

[2] A. Avila, J. Kahn, M. Lyubich, W. Shen, Combinatorial rigidity for unicritical polynomials, preprint IMS at Stony Brook, \#5, 2005. To appear in Annals of Math..

[3] D. Cheraghi, Combinatorial rigidity for some infinitely renormalizable unicritical polynomials, preprint IMS at Stony Brook, \#7, 2007.

[4] A. Douady, J. H. Hubbard, On the dynamics of polynomial-like mappings, Ann. Sci. École Norm. Sup. 18 (1985), 287-343.

[5] F. P. Gardiner, N. LAKic, Quasiconformal Teichmüller theory, Mathematical Surveys and Monographs 76, Amer. Math. Soc., 2000.

[6] J. KAHN, A priori bounds for some infinitely renormalizable quadratics: I. bounded primitive combinatorics, preprint IMS at Stony Brook, \#5, 2006.

[7] J. Kahn, M. Lyubich, Local connectivity of Julia sets for unicritical polynomials, preprint IMS at Stony Brook, \#3, 2005. To appear in Annals of Math..

[8] J. KAHN, M. LyUbich, Quasi-additivity law in conformal geometry, preprint IMS at Stony Brook, \#2, arXiv:math.DS/0505191v2, 2005. To appear in Annals of Math..

[9] M. Lyubich, Dynamics of quadratic polynomials. I, II, Acta Math. 178 (1997), 185247, 247-297.

[10] C. T. McMullen, Complex dynamics and renormalization, Annals of Mathematics Studies 135, Princeton University Press, 1994.

[11] J. Milnor, Local connectivity of Julia sets: expository lectures, in The Mandelbrot set, theme and variations, London Math. Soc. Lecture Note Ser. 274, Cambridge Univ. Press, 2000, 67-116.

[12] J. Milnor, Periodic orbits, externals rays and the Mandelbrot set: an expository account (Géométrie complexe et systèmes dynamiques, Orsay, 1995), Astérisque 261 (2000), 277-333.

(Manuscrit reçu le 10 janvier 2007; accepté, après révision, le 19 décembre 2007.)

\footnotetext{
Jeremy KAHN

Stony Brook University

Mathematics Department

Stony Brook, N.Y. 11794-3651, U.S.A.

E-mail: kahn@math.sunysb.edu

Mikhail LyUBICH

Stony Brook University

Mathematics Department

Stony Brook, N.Y. 11794-3651, U.S.A.

E-mail: mlyubich@math.sunysb.edu
}

$4^{\mathrm{e}}$ SÉRIE - TOME $41-2008-\mathrm{N}^{\mathrm{o}} 1$ 